\title{
IoT Technologies for Livestock Management: A Review of Present Status, Opportunities, and Future Trends
}

\author{
Bernard Ijesunor Akhigbe 1,2,*(D), Kamran Munir ${ }^{2,3}$, Olugbenga Akinade ${ }^{3}$, Lukman Akanbi ${ }^{1,3}$ \\ and Lukumon O. Oyedele ${ }^{3}$ (D) \\ 1 Department of Computer Science \& Engineering, Obafemi Awolowo University, Ile-Ife 220282, Nigeria; \\ Lukman.Akanbi@uwe.ac.uk \\ 2 Department of Computer Science and Creative Technology, University of the West of England, \\ Bristol BS16 1QY, UK; Kamran2.Munir@uwe.ac.uk \\ 3 Big Data Enterprise and Artificial Intelligence Laboratory, University of the West of England, \\ Bristol BS16 1QY, UK; Olugbenga.Akinade@uwe.ac.uk (O.A.); L.Oyedele@uwe.ac.uk (L.O.O.) \\ * Correspondence: biakhigbe@oauife.edu.ng or benplus1@gmail.com
}

Citation: Akhigbe, B.I.; Munir, K.; Akinade, O.; Akanbi, L.; Oyedele, L.O. IoT Technologies for Livestock Management: A Review of Present Status, Opportunities, and Future Trends. Big Data Cogn. Comput. 2021, 5, 10. https://doi.org/10.3390/ bdcc 5010010

Received: 11 January 2021

Accepted: 19 February 2021

Published: 26 February 2021

Publisher's Note: MDPI stays neutral with regard to jurisdictional claims in published maps and institutional affiliations.

Copyright: (c) 2021 by the authors. Licensee MDPI, Basel, Switzerland. This article is an open access article distributed under the terms and conditions of the Creative Commons Attribution (CC BY) license (https:/ / creativecommons.org/licenses/by/ $4.0 /)$.

\begin{abstract}
The world population currently stands at about 7 billion amidst an expected increase in 2030 from 9.4 billion to around 10 billion in 2050. This burgeoning population has continued to influence the upward demand for animal food. Moreover, the management of finite resources such as land, the need to reduce livestock contribution to greenhouse gases, and the need to manage inherent complex, highly contextual, and repetitive day-to-day livestock management (LsM) routines are some examples of challenges to overcome in livestock production. The Internet of Things (IoT)'s usefulness in other vertical industries (OVI) shows that its role will be significant in LsM. This work uses the systematic review methodology of Preferred Reporting Items for Systematic Reviews and Meta-Analyses (PRISMA) to guide a review of existing literature on IoT in OVI. The goal is to identify the IoT's ecosystem, architecture, and its technicalities-present status, opportunities, and expected future trends-regarding its role in LsM. Among identified IoT roles in LsM, the authors found that data will be its main contributor. The traditional approach of reactive data processing will give way to the proactive approach of augmented analytics to provide insights about animal processes. This will undoubtedly free LsM from the drudgery of repetitive tasks with opportunities for improved productivity.
\end{abstract}

Keywords: IoT technologies; IoT ecosystem and architecture; artificial intelligence; big data; cloud computing; 5G nexus; PRISMA methodology

\section{Introduction}

The world population currently stands at about 7 billion. This number is bound to increase according to projections between 9.4 billion and 10.1 billion in 2030 and 2050, respectively [1,2]. In this rapidly growing world population, the upward demand for animal food (UDfAF) is inevitable. On the one hand, the inherently complex nature of livestock farming, the gradual reduction in the global workforce, and the increasing production cost of food [2] would increasingly make it difficult to meet the UDfAF. On the other hand, there is the management of finite resources like land, the need to reduce livestock contribution to greenhouse gases, and the need to manage highly contextual and repetitive day-to-day routines in livestock management (LsM) as additional challenges to overcome in livestock production. With this burgeoning population, more challenges are likely to join this list.

Interestingly, the world is at the cusp of witnessing another phenomenon that will rely on IT. Named "the fourth wave of human life" [3]. This fourth wave or "cyber-age" would usher on the Internet of Things (IoT) through which almost everything would be controlled [3-5]. Progress regarding its emergence has culminated in the birth of disruptive 
and retrofit technologies such as sensors, actuators, and other IoT-compliant technologies. These sensors and actuators are retrofittable. In the information and communication environments, networks, objects, or "Things" become smart and evolve into IoT technologies (IoTTs) that interact with each other and act autonomously. Smart farming in the literature is inclusive of precision livestock farming (PLF) that is conceptualized as the farm management approach that support the use of IoT [2]. In [6-8], as in other related literature, the role of IoT in the management of livestock to overcome the type of challenges (and more) highlighted earlier regarding animal food production is not clear. Additionally, the barriers to overcome the adoption of IoT has not been addressed in the literature regarding LsM. The purpose of this paper is to fill this gap using a systematic review methodology.

There is a significant body of literature on IoTT from several sources, such as wikis, blogs, proceedings from conferences, forum posts, journal papers, etc. For example, [2] used the Preferred Reporting Items for Systematic Reviews and Meta-Analyses (PRISMA) methodology to survey the use of IoT within the agriculture economics context with a specific focus on hydroponics. In comparison with [2], this study focuses on LsM instead of hydroponics. While the survey carried out by [8] was about the next generation of PLF technologies, [7] reviewed the aspect of commercializing PLF technologies, and [6] apprised PLF regarding the technologies that are available for the swine industry. In contrast to [6-9], this study emphasises the possibilities and opportunities for LsM when IoTT work with technologies such as the ones re-counted in [6-9]. This paper used the systematic literature survey (SLS) methodology_PRISMA to survey existing literature to identify the role of IoTT in LsM and its adoption barrier in LsM.

This paper makes its contribution by explaining the model of IoT. This model is typified as illustrated in Figure 1. The diagram depicts the use of a multi-layer smart method to manage livestock.

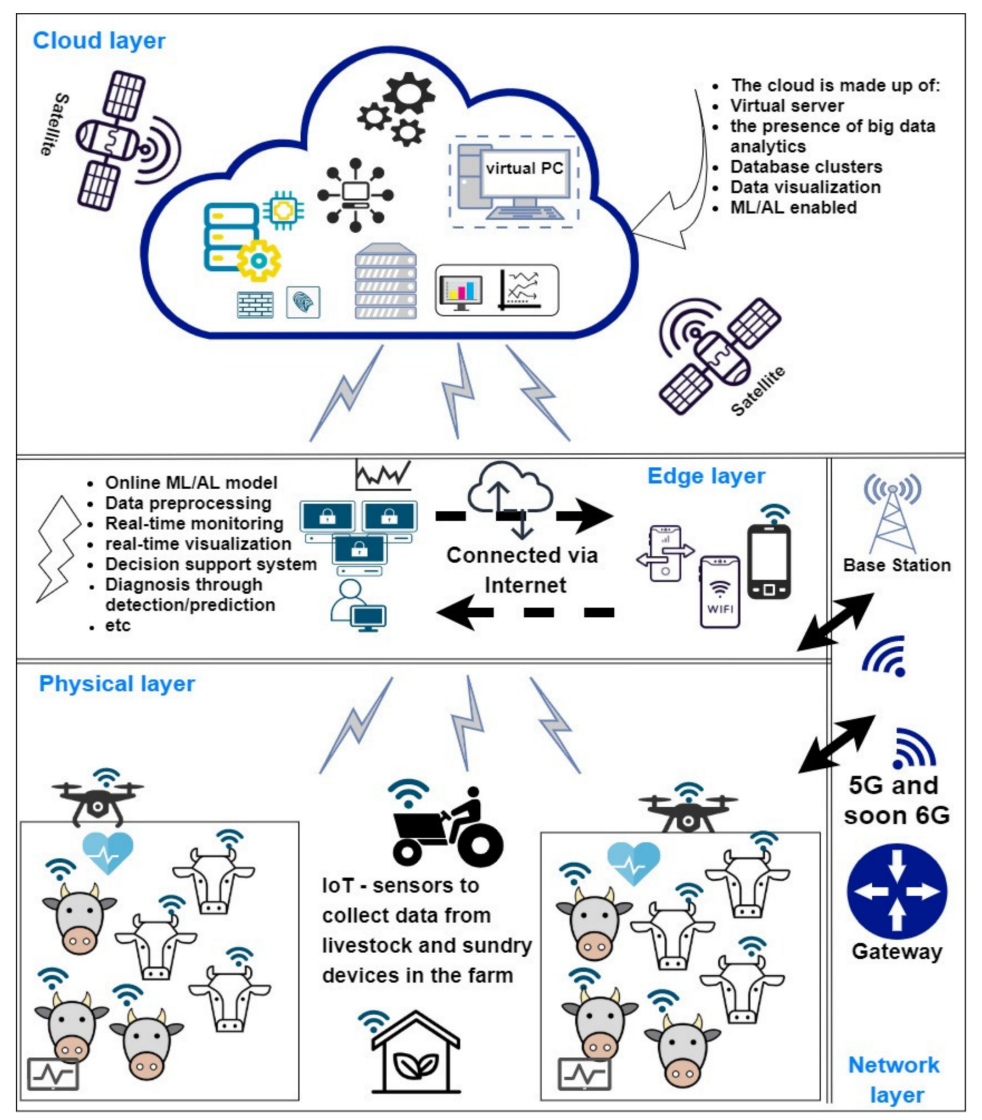

Figure 1. Illustration of a multi-layer smart Internet of Things (IoT) approach for livestock management. 
The smart method is made up of four layers, namely the cloud, edge, physical, and network layers. The cloud layers is made up of the virtual server with the presence of big data analytics. There is also the presence of database clusters with potential for data visualization and enabled machine learning (ML) and artificial intelligence (AI) potentials. The edge layer is where the users' computational activities are enabled with the existence of ML and AI models and data processing capability, among other features (See Figure 1). The physical layer is the layer responsible for enabling the sensors that are retrofitted into animal far equipments and placed strategically on the animals to collect data and make them available computation and sundry feedback. The network layer performs the function of offering the gateway to allow data communication and sundry connectivity. This is where the $5 \mathrm{G}$ and the soon coming $6 \mathrm{G}$ network operates to allow a faster Internet.

The IoT ecosystem is also presented along with its driver and enabling technologies. The context of disruptive technology and the five-layered IoT architecture instead of the three layers identified in [2] are presented as contributions in this paper regarding IoTTs management potentials (i.e., role) in LsM. Emphasis was also particularly given to the barriers that could hinder the adoption of IoTT in LsM that [2,6-8] and others excluded in their surveys. The elements of IoT (i.e., features) are highlighted as the key factors that make IoT uniquely positioned to drive LsM in the 21st century. On the one hand, the review offers information, which stakeholders can rely on to understand IoT's use within the context of LsM to encourage further research towards better services in LsM. On the other hand, the methodology of PRISMA offers a benefitting and useful tool to study the critical role of a favoured method in a review that seeks to enlighten and encourage the rethinking of classic methods. This paper also sheds light on the state of IoT knowledge to clarify possible contradictions and pioneered the stimulation of broad unanimity (i.e., consensus) regarding the use of IoT for LsM.

This review is structured as follows. Section 2 presents IoT concepts, and Section 3 shows the review methodology used for the survey. Section 4 introduces IoT as a cyber physical system and example algorithms to drive its potentials. Section 5 presents the application of IoT and its usefulness in LsM. Section 6 discusses IoT's opportunities for LsM. Section 7 summarises some possible future directions, the role of IoTs, and the barriers to its adoption in LsM. In Section 8, the cost implication of using IoTs to manage livestock is discussed, while Section 9 concludes the paper.

\section{Concepts}

It is important to enlighten on the basic concepts of IoT to help understand the role and use of IoT in LsM in this 21st century. Hence, IoT as a paradigm, its ecosystem, and architecture are presented in this section to indicate its role regarding the possible solution(s) it will provide in LsM. IoT's supporting and main technologies (i.e., the technologies that enable it to be a paradigm) as well as its technicalities in the livestock context are also presented.

\subsection{IoT Ecosystem and Players}

In the deployment of IoTTs concerted technical assistance (CTA) is required. This brought about the existence of IoT ecosystem (IoT-E) (see Figure 2). The IoT-E is a conception that captures the key players that are responsible for providing the CTA. The result of a desk research review of the literature on IoT-E of which a summary is captured in Figure 2a showing that the ecosystem is made up of five key (industry) players. These players make both technical and device contributions in relation to the technical needs that are captured in the IoT five-layer architecture shown in Figure $2 \mathrm{~b}$. The big circle in Figure 2a seeks to show the possible areas of IoT application generally. The area of application is, however, not limited to what is listed within the big circle, since as more disruption occurs in the use of IoT, more areas are bound to be included. Example of some key players are Microsoft, IBM, Amazon, and Google, etc. In the depicted IoT-E in Figure $2 \mathrm{a}$, these players fall within two types of players-application and solution provides 
(A\&SP) and developers of software platforms (DoSP)-because of the type of services they offer within the ecosystem. These services are provided at affordable costs with minimal technological barriers and within simplified IoT application development processes [10]. The other three key players' categories are: developers of hardware platforms (DoHP), developers of network technology (DoNT), and users and consumers (U\&C). These services are captured using contribution in Figure 2a. IoT-E is meant to encourage and strengthen standards globally and make IoT easy to adopt for use at different scale.

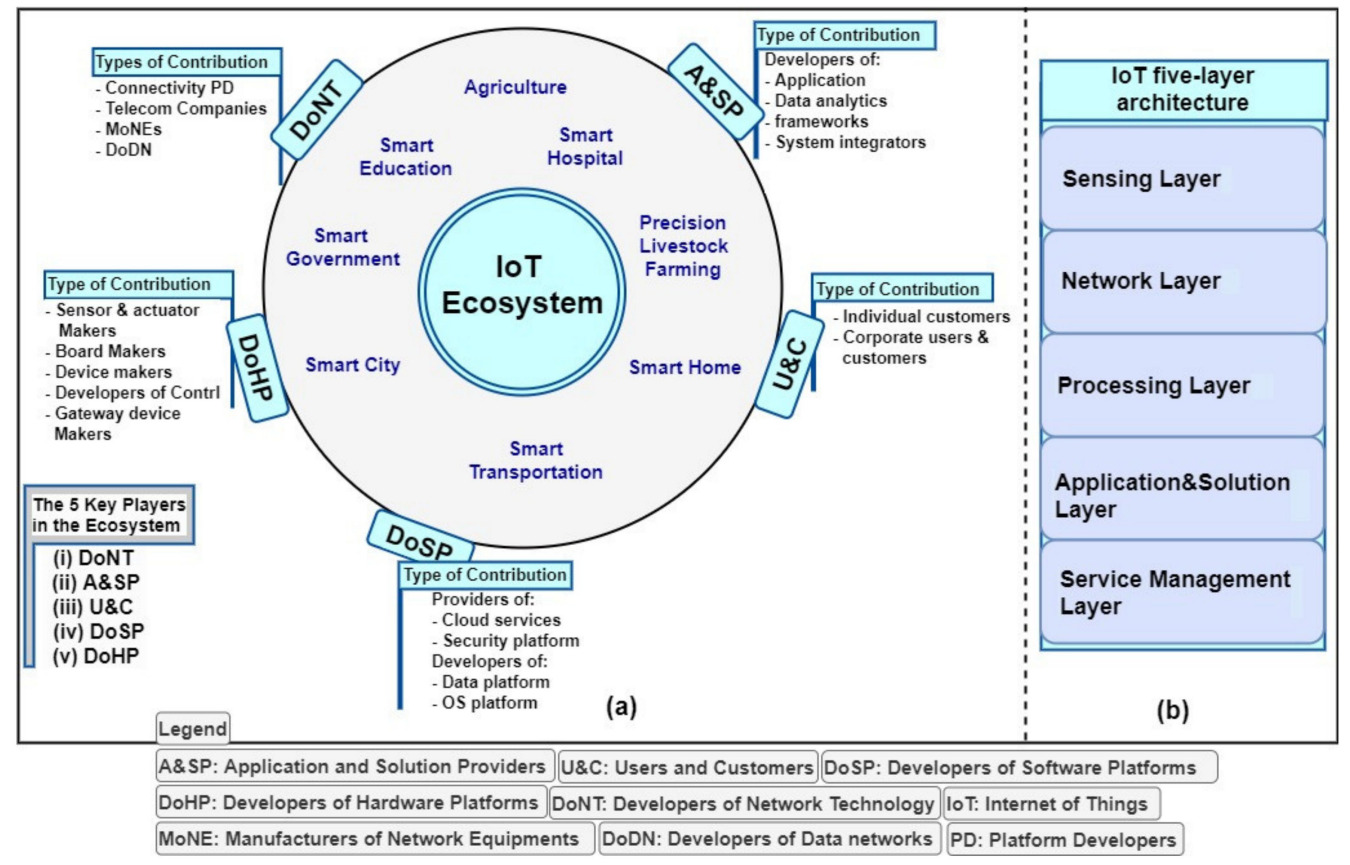

Figure 2. (a) A typical IoT ecosystem with (b) architecture.

The IoT architecture (see Figure 2b) helps stakeholders (players) to see what services and possible solutions are available for easy adoption. It also highlights the possible services key players in the IoT-E could provide. This makes the IoT-E interesting in that it has a lot of service components to focus on, which, however, makes it difficult for all the services to be provided by a single player (i.e., company) in the IoT-E. For example, cloud based solution(s) will be provided by the key players

In the DoSP, key player category is used in the processing layer. The DoHP key player category will be responsible for providing gateway device solutions to satisfy the need for devices to handle raw data transmission and processing issues [11]. It is worth noting that there are several players in each key player category. For example, in the A\&SP key player category, there are industry partners and collaborators involved in providing IoT user-based application; some are involved in data analytics solution, while others provide frameworks and solution for system integration. Similarly, in the U\&C key player categories, customer experiences are enhanced through service provisioning that includes billing, logistics, customer support, and marketing at B2B scale. Security platforms are built within the DoSP player category into IoT solutions either at the hardware, service, firmware, and software level. This way it is possible to stay ahead of any threat [10-12].

To realise intelligent-based tasks (I-bTs), IoTTs would communicate and share information [13] with every single "thing" if they have attribute and unique identity. In real-life, these I-bTs include perception, positioning, tracking, monitoring, identification, and management $[13,14]$. These are basically the same tasks that are entailed in livestock management (LsM). Based on these entailments, IoTTs would perform these I-bTs in the management of livestock. This implies that livestock management would be done more intelligently to maximize livestock yield potentials while minimizing the challenges of rearing them [15]. LsM demands animal-specific attention (A-sA). During animal maturation 
for instance and other sundry individual animal developmental comportments A-sA are required. This underscores the need for eyes to be on ground to monitor, track as well as identify each animal on a one on one basis to cater for their uniqueness even within the same species. These activities and processes are regimented and quite complex and do not succeed with a one size fit all, hence burdensome and arduous, approach [16,17]. However, breeding practices such as knowing when an animal is ready to mate and give birth and the prevention of the loss of a new calf will be improved through the sensing potentials of IoT. The wherewithal to respond to the foregoing is evident in the IoT-E, since there are players that are already positioned to respond regarding the provision of solution to drive each entailment.

\subsection{The Paradigm of IoT}

IoT is a paradigm that allows numerous sensing and actuating devices to interconnect through the Internet, as such data are collected, shared, and analyzed for innovative application. As the next evolution of the Internet, IoT will make it possible to collecttransmit-distribute, sense, and analyze data on a massive scale. Unlike the current Internet, the IoT's Internet will be pervasively inclusive and allow ubiquitous computing with layers of interconnectivity and interactions. As an all-inclusive interaction platform that uses both hardware and software, data will be its common denominator as depicted in Figure 3. Data generation will also be the most critical part of its process. The strength of IoTTs will drive this paradigm. By sensing, identification of "things" and the enforcement of communication and interaction with "things" will be done better than humans. This is because unlike humans, IoTTs will not be limited in context and in the number of tasks to perform. The IoT's goal is to collect data in huge volume from different contexts and domains and support their processing [18]. These processed data will be used to make informed decision and take valuable actions to stay competitive with improved efficiency [19].

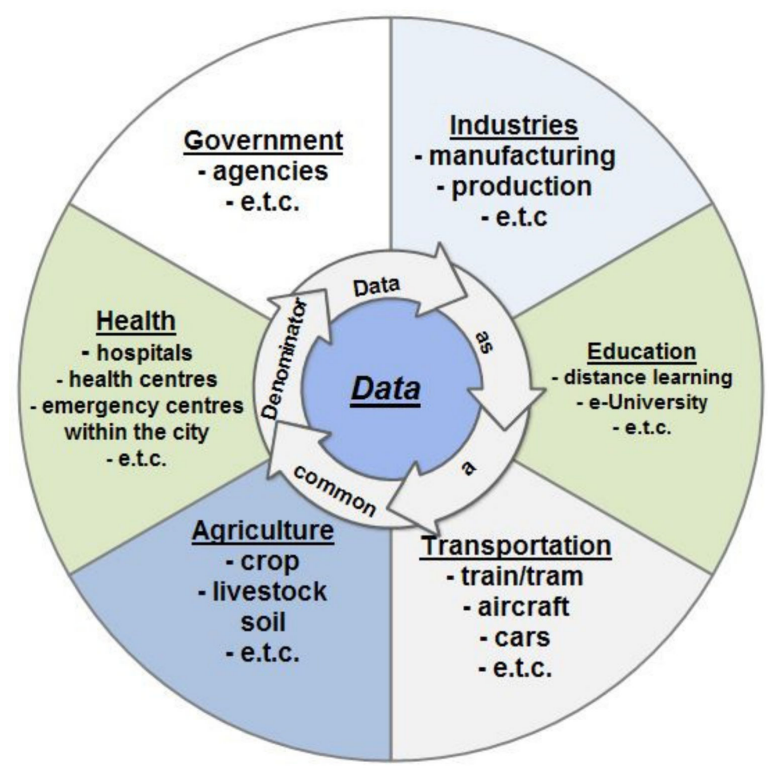

Figure 3. Data as a common denominator and possible integrator with sundry domains.

A major characteristic of IoT as a paradigm is proliferation. Based on the literature search, different use of the concept of IoT were identified as the reason why it has many aliases, which also reveal its area of specific application [20-22]. Some of such aliases are Internet of Underground Things (IoUT), Industrial Internet of Things (IIoT), Livestock Internet of Things (LIoT), Internet of Space Things (IoST), Internet of Underground Rail (IoUR), Agricultural Internet of Things (AIoT), and Internet of X-Things (IoXT), etc. [22-24]. While some are still at the proposal stage, some are at the prototype phase with other 
awaiting full implementation. There are different stakeholders with varied interest in IoT that include the scientific research community, sales strategists, and even marketing agents. Part of what has advanced the different perspectives and visions that are evident about the paradigm of IoT is the varied and continuous interest in IoTT. Similarly, there is also the continuous evolvement of IoT thematic, in which proliferation is due to the incessant change in the ideas and technologies that drives IoTT [25]. Interestingly, despite these varied areas of application data as earlier mentioned, it will still remain the common interest and denominator for the different stakeholders. This will motivate the collection of domain specific data with context given attention as a major reason for the data collection, thus necessitating the customisation of IoT to suit such purpose.

With the paradigm of IoT data will be generated on a large scale and such data will inevitable exhibit the Incompleteness and Heterogeneity (I\&H) as well as privacy and timeliness data characteristics. Additionally, this data generation will increase rapidly. While timeliness will focus on data acquisition rate, privacy will deal with the protection of sensitive data attributes and personal identities during analytic processes. The focus of I\&H will be using big data technologies despite the possibility of missing out (some) attributes in the face of data heterogeneity. How stakeholders would cope and handle these data characteristics will determine the proportion and scale of IoT-based innovations. In the literature, this has been identified as capable of driving the creation of innovative opportunities to resolve challenges with novel innovations. Beside these novel innovations digital convergence will thrive. For example in [26], computing, IoT big data storage and processing, and data abstraction were identified as potential drivers of digital convergence. This has implications, which are development at several different fronts with the most relevant being cloud computing and the 5G network. While cloud computing is summarily primed to decentralise the power of computing along networks with guaranteed reliability and response time, the $5 \mathrm{G}$ network will facilitate access to application and services in the cloud with guaranteed quality of experience [27].

\subsection{IoT and Its Architecture and Enabling Technologies}

IoT has the potential to effect transformation wherever it is deployed for assistance. However, this is not without a good understanding of its architecture and enabling technologies [28,29]. As a bespoke technology, this understanding is needed and can be realized through the knowledge of its architecture and enabling technologies. This section, therefore, discusses this in terms of IoT's functionalities within the context of LsM.

\subsubsection{IoT Architecture}

IoT's implementation require the use of several devices and applications (D\&Apps) [30]. This makes it tricky to manage a typical IoTT implementation. Some of the issues with this include the problem of localization (i.e., what D\&Apps should do what in a functional area), and how to accommodate and maximize the emergence of newer techniques and technologies when the need arises. As much as possible, these challenges should be managed to allow the creation of innovative services, hence the use of an IoT architecture (IoTA). Although, the IoT ecosystem is responsible for providing the technological platforms to creatively implement what is entailed in a typical IoT architecture (IoTA); the IoTA's importance is still overreaching, since it provides the wherewithal to maneuver the tricky management of the characteristic IoTT implementation. The absence of a consensus architecture to implement the IoT in sundry area of application in the literature still makes this maneuvering a lot difficult. What obtains is the use of a multi-layered approach [31] that fosters a function-oriented perspective (FoP) of IoTT application. The FoP makes it possible to drop and/or add functions when the need arises. In [10], this was explained as the capability to continuously update, commission, and decommission IoT assets and services. This current flexibility of accommodating newer technique(s) that IoTA offers makes it imperative to consider IoT as a huge resource to manage livestock. 
The spread of IoT is inevitable and has resulted in the proliferation of architecture with various novel standards with interoperability being one of its resultant challenges [32]. The attempt to resolve this yielded the arrowhead framework (AHF), which is one of the several solutions to solve the problem of interoperability and, by extension, integration [32] The AHF has been useful for the discovery of operation and inclusion of sundry services within the service-oriented architectures (SOA). This potential makes it possible to integrate proprietary and legacy systems, thus making AHF an important framework to consider in LsM. For example, AHF has been used to achieve the acquisition of data from sensors within the context of a typical industrial environment. The AHF as an architecture has service delivery orientation, which is, hence, considered a viable option to guide the implementation of systems that are tailored for different purposes. Its ability to utilize the SOA comes with benefits in respect of security, good use of engineering time, latency, and scalability among others. AHF operation is possible through reliance on three systemsthe Service-Registry, Orchestration, and Authorization System. These are the core and mandatory systems that make it possible for AHF to achieve its basic functionalities [32,33]. There are other open-source frameworks (or protocols) like AHT such as Eclipse, FIWARE, BaSyx, and IoTivity, which details are found in the literature e.g., [34] for interested redears.

Usually, systems that are arrowhead compliant do collaborate, and when it happens, they become a system-of-systems (SoS). SoS also do collaborate and are built from several subsystems at the level of application based on SOA patterns with AHF offering interconnectivity requirements. Thus, AHF naturally enables complex solutions. Arrowheads are reconfigurable for all kinds of architectures and manages to fix and cover all security gaps in applications with real-time configuration. Detailed information about AHF, SoS, SOA, and how they converge to address issues of interoperability and interconnectivity while ensuring quality of service, service consumers, and producers versus late binding and loose coupling, etc., are out of the scope of this review abound in the literature $[32,35,36]$. Nevertheless, there are different types of architecture and framework proposed to implement different aspects of the IoT [37,38]. Tables 1 and 2 contain a summarized comparison of architecture and framework based on their contributions to the deployment of IoT. In Table 1, the comparison is presented based on the standard practice of classifying IoT architecture, which is the layered, domain-specific, and industrial architecture in orientation [38]. In the comparison presented in Tables 1 and 2, while architecture is intended to capture IoT devices and implementation scenario, the framework captures a summary of solution proposed to address operational concerns with IoT deployment. This comparison followed the practice in $[27,39]$.

The nature of LsM makes it a domain to always try new methods in managing the many and sometimes inexplicable pattern of animal behaviours. The IoTA is significant in this regard because of its flexible and accommodative nature to allow the adoption of new technological practices. This has implications for LsM, especially in the context of the IoT. The consequence is the flexible application of newer techniques such as the $\mathrm{AI}$ in confluence with IoT. IoT-AI-driven technology, for example, when built on algorithms, can analyze and learn from data to improve over time by adjustments with no human intervention. The IoTA support in the deployment of IoT-AI-driven technology will dramatically effect incremental improvements against what has been known over the last century [40,41].

\subsubsection{IoT's Enabling Technologies}

In [42], the use of a middleware to resolve the challenge of heterogeneity when deploying IoT's enabling technologies was highlighted. These enabling technologies are enablers of real-time communication in [43]. The 5G mobile network will drive a fast Internet that is better than what it is now. This role will allow the broader use of IoT sensors and actuators [44]. Unlike the previous generation network, 5G will rely on the potentials of its features to play this role. The features include the enhanced mobile broadband (eMBB), massive machine-type communications (mMTC), and ultra-reliable and low-latency communications (URLLC). 
Table 1. A summarized comparison of IoT architecture based on its layered, domain-specific, and industrial orientation.

\begin{tabular}{|c|c|c|c|}
\hline Architecture Type & Objective/Features & Comment & Cit. \\
\hline Layered & $\begin{array}{l}\text { To highlight the services to be delivered } \\
\text { with the intention of addressing them } \\
\text { separately and emphasizing integration } \\
\text { before performance as a system. } \\
\text { At the moment, the IoT architecture has } \\
\text { been extended from the initial: } \\
\text { (i) Three layers (of application, network, } \\
\text { and perception layers) through five } \\
\text { layers (of business, application, } \\
\text { processing, network, and perception } \\
\text { Layers) to seven layers (of collaboration } \\
\text { and processes, application, data } \\
\text { abstraction, data accumulation, } \\
\text { Edge-Fog computing, connectivity, and } \\
\text { physical devices and ontrollers layers). }\end{array}$ & $\begin{array}{l}\text { - It offers the prospect of conceptualizing } \\
\text { IoT scenarios within levels of complexity } \\
\text { and integration of several types of } \\
\text { technologies and services so as to } \\
\text { manage the complexity of IoT systems. } \\
\text { The layered approach allowed the } \\
\text { components that are required for the } \\
\text { continuous, coherent, and uniform } \\
\text { adoption of IoT. } \\
\text { It helps to track the growth in IoT } \\
\text { research primarily based on the } \\
\text { requirements and needs of corporations. } \\
\text { By this classification using the layered } \\
\text { approach, what framework(s) and how } \\
\text { to implement them and sort out issues } \\
\text { are understood. } \\
\text { It also helps to understand IoT devices } \\
\text { that are enabled to provide collaborative } \\
\text { and cooperative services. }\end{array}$ & [38]. \\
\hline \multirow[t]{2}{*}{$\begin{array}{l}\text { Domain- } \\
\text { Specific }\end{array}$} & $\begin{array}{l}\text { - Health-based IoT architecture was } \\
\text { proposed and composed of two sub-parts: the } \\
\text { part that handles data concerns and the one } \\
\text { that handles security issues between the fog } \\
\text { and cloud layer. }\end{array}$ & $\begin{array}{l}\text { The scenario covered include: } \\
\text { - } \quad \text { Different target groups; } \\
\text { The monitoring of vital signs and health } \\
\text { status with the provision of statistic and } \\
\text { predictive analysis; } \\
\text { Meet critical requirements (e.g., } \\
\text { real-time processing with high accuracy } \\
\text { and availability). }\end{array}$ & {$[45,46]$} \\
\hline & $\begin{array}{l}\text { The other domain-specific architecture } \\
\text { include (but are not limited to this list) that of } \\
\text { Internet of vehicle, agriculture, smart city, and } \\
\text { manufacturing, etc. }\end{array}$ & $\begin{array}{l}\text { These architecture provide a solid backbone to } \\
\text { deploy intelligent solutions in these domains. }\end{array}$ & [45-47] \\
\hline $\begin{array}{l}\text { Industrial/ } \\
\text { commercial }\end{array}$ & $\begin{array}{l}\text { Industry-basedIoT architecture has } \\
\text { been proposed with consumer-based } \\
\text { systems orientation. } \\
\text { It is based on interconnected intelligent } \\
\text { devices of industry proportios and the } \\
\text { framework to use IoT-focused } \\
\text { digitalization. }\end{array}$ & $\begin{array}{l}\text { Applicable in many scenarios with } \\
\text { industrial inclination like livestock } \\
\text { farming. } \\
\text { The architecture framework assumes the } \\
\text { viewpoints of usage, business, function, } \\
\text { and implementation }\end{array}$ & {$[46,47]$} \\
\hline
\end{tabular}

- Generally:

IoT Architectures seeks to shed light on what technology to incorporate into IoT scenarios as the need arises. This can be either bidirectional and/or unidirectional with expanding use to several diverse real-life scenarios. 
Table 2. Presents a summarized comparison of IoT framework.

- The framework did not fully capture the requirements for resilience to handle multi-dimensional disturbances.

- A double 3-dimensional framework that helps to explore the adoption of networking technologies such as 5G, M2M communication, software-defined networking, computing in I-IoT, cloud, hybrid cloud, and edge computing platforms was presented.

- $\quad$ The perspective of Cyber Physical System was adopted with a focus on networking, control, and computing.
- The techniques to improve the resilience of control system disturbances are needed.

- Network routing optimization and deployment issues need further attention.

- Low-latency requirements and the seamless integration of edge computing and industrial IoT are limited/excluded.
- The work informed on the possibility of exercising system control using industrial devices such as actuators, sensors, retro-fitted equipment, etc., on a massive scale for productive gains.
The survey highlighted the following:

- IoT framework guide the deployment of IoT applications.

- $\quad$ The success of IoT applications depends IoT framework and the ecosystem characteristics of the framework.
- Although the security of IoT frameworks was surveyed, limited discussion provided to address those security challenges.
- The work supports the claim that there are several IoT frameworks and conceptualises its essence.
- The knowledge of a framework's entry barriers to the market that include licenses, accessibility, industry support, bad accessibility of code and resources, and the lack of information and specifications was considered an important aspect of comparing its technical characteristics. However, other entry barriers such as lack of trust in the community, corporate image, etc., were not fully considered.
- The work highlighted gains of deploying large scale SoS flexible and scalable devices and systems. A

framework-based solution was advocated, which was nevertheless difficult to select due to the rising amount of accessible platform and frameworks. To fill this gap features of some IoT key framework were investigated to aid the simplification of selecting a suitable framework.
- $\quad$ IoT-SE research is sparse and still at its infancy. Further study is useful regarding the development of intelligent/efficient IoT search.

- Identified the challenges of retrieving IoT data and finding IoT devices and their twine challenge of the need for timely and efficient query processing as the consequence of the novel ecosystem for actuation and sensing created by the IoT paradigm on an exceptional scale.

- To fill this gap, the work proposed a framework that is generic for the IoT search engine's (IoT-SE) deployment with a naming service. The work also outlined research solutions for developing intelligent and efficient IoT-SE by addressing an aspect of query processing and applied a neural network architecture to deliver predictive query retrieval based on multiple targets.
- In the current work the aspect of semantic search was not/partly considered.

- Additionally, both privacy and security issues still remain an open area of research regarding search in IoT, which was beyond the research focus of the work.

- Other aspects of research to consider include the optimisation of (i) query processing, (i) data retrieval, and (ii) data discovery and the handling of redundancies.
- The work highlighted the importance of frameworks in the deployment of IoT paradigm in an industrial scale.
- The work supports the discourse presented in the review that the advances in ML and obvious potentials when in synergy with DL-based predictive ability will improve IoT use as a resource to play a management role in LsM.
- The use of IoT framework to implement data acquisition has been recognised in the literature.

(i) The non-exposure to extensive IoT network devices motivated a proposed data acquisition IoT-permitted framework.

(ii) Secondly, in similar research, a framework to develop a data acquisition system that integrates with robotic technology and web-enabled technologies.
- $\quad$ Issues of syntactic and semantic interoperable that impede data acquisition and cybersecurity concerns were not given attention.

- $\quad$ Other issues that need to be address include authentication methods and security concerns that obstruct the deployment of services in the cloud. The work did not consider real-life case studies' performance issues.
- It can be concluded that a framework using IoT devices and related networks can be used to develop a platform-neutral, cost-effective, and scalable data acquisition system for smart IoT-oriented innovations.

- $\quad$ The possibility of deploying a framework to realize the automatic generation of software components and resultant repositories to be deployed in the cloud was highlighted.
- $\quad$ The work identified privacy protection and security requirements as well as the need to maintain processing efficiency to conserve the consumption of battery power as research gaps.

- A two-tier privacy-preserving data inference solution framework.
- $\quad$ Federated learning and blockchain techniques are considered for incorporation in the future to improve privacy and high-accuracy data inferencing system.

- $\quad$ Additionally, the work did not show a proof of work and concept validation with appropriate use cases.
- The framework will be useful in deploying IoHT devices to monitoring zoonotic diseases outbreak.

- The conservation of IoT devices' battery deployed in a livestock farm. 
The eMBB, mMTC, and URLLC are tantamount to three service-based usage scenarios. In the eMBB scenario, high data rates and user density with improved latency and traffic capacity for limitless hotspots and coverage are possible. Whereas, in the mMTC scenario, data rates and power consumption are low and the numbers of devices are easily connected simultaneously; in the URLLC scenario, mission and safety critical communication concerns are addressed to ensure reliability and responsiveness [53]. Based on the features of 5G limitations such as high energy consumption, end-to-end delay, inability to support high density of devices, etc., that characterize $3 \mathrm{G}$ and $4 \mathrm{G}$ networks have been removed. This same capability will make it possible for IoT to play its role in the management of livestock where sundry service-based usage scenarios are extensive. Aside from the advantages offered by $5 \mathrm{G}$ based on its features, there are other benefits such as security, mobility support, economy of scale, virtualization and softwarization, etc. Details of the technical requirements and use cases that allow infrastructure retrofit and process monitoring, etc., that are out of the scope of this review are available in related texts such as [28,43,53-55].

IoT's enabling technologies underlie its fabric [25]. These technologies support IoT's implementation and deployment. The list of these technologies are likely going to increase, since IoT is still at its emerging phase [56]. In [57], the relationship between IoT and its emerging technologies was highlighted and appraised to interrogate what IoT requires to deal with privacy and cyber physical system concerns. These enabling technologies are presented to show where their support lies in the role IoT and its functionalities seek to play in the management of livestock $[21,58]$. Table 1 shows some of the relevant enabling technologies and their functionalities using IoT elements: identification (Id), sensing (Ss), communication $(\mathrm{Cm})$, computation $(\mathrm{Cp})$, services $(\mathrm{Sv})$, and semantics $(\mathrm{Sm})$ (see Table 3$)$. In Table 3, different categories of IoT-enabling technologies that are capable of supporting its functionalities towards low-cost IoT solution in LsM are presented. 
Table 3. Type of enabling technologies for IoT and based on its features.

\begin{tabular}{|c|c|c|c|c|c|c|c|c|c|c|c|c|c|c|c|}
\hline \multirow{2}{*}{ Enabling Technologies } & \multicolumn{7}{|c|}{ IoT's Elements } & \multirow{2}{*}{ Enabling Technologies } & \multicolumn{7}{|c|}{ IoT's Elements } \\
\hline & Id & Ss & $\mathrm{Cm}$ & $\mathrm{Cp}$ & Sv & Sm & Cit & & Id & Ss & $\mathrm{Cm}$ & $\mathrm{Cp}$ & Sv & $\mathrm{Sm}$ & Cit \\
\hline WiFi & $x$ & $x$ & $\sqrt{ }$ & $x$ & $x$ & $x$ & \multirow{5}{*}[28,40,41]{} & Arduino & $x$ & $x$ & $x$ & $\sqrt{ }$ & $x$ & $x$ & \multirow{5}{*}{ [40] } \\
\hline Bluetooth & $\mathrm{x}$ & $\mathrm{x}$ & $\sqrt{ }$ & $x$ & $x$ & $x$ & & Phidgets & $x$ & $x$ & $x$ & $\sqrt{ }$ & $x$ & $x$ & \\
\hline 13E-802 & $\begin{array}{l}\mathrm{X} \\
\mathrm{x}\end{array}$ & $\begin{array}{l}x \\
x\end{array}$ & $\sqrt{ }$ & $\underset{x}{X}$ & $\mathrm{X}$ & $\begin{array}{l}X \\
x\end{array}$ & & Intel Galilio & $\mathrm{x}$ & $\mathrm{x}$ & $x$ & $\sqrt{ }$ & $x$ & $\mathrm{x}$ & \\
\hline Z-wave & $\begin{array}{l}x \\
x\end{array}$ & $\mathrm{X}$ & $\sqrt{ }$ & $\mathrm{x}$ & $\mathrm{X}$ & $\mathrm{x}$ & & ID-Related & $\mathrm{x}$ & $\mathrm{x}$ & $\mathrm{x}$ & $\mathrm{x}$ & $\sqrt{ }$ & $\mathrm{x}$ & \\
\hline LTE-A & $\mathrm{x}$ & $\mathrm{x}$ & $\sqrt{ }$ & $\mathrm{x}$ & $\mathrm{X}$ & $\mathrm{x}$ & & Smart sensors & $\mathrm{x}$ & $\sqrt{ }$ & $x$ & $\mathrm{x}$ & $x$ & $\mathrm{x}$ & \\
\hline RFID & $x$ & $x$ & $\sqrt{ }$ & $x$ & $x$ & $x$ & [28] & Wearable sensors & $x$ & $\sqrt{ }$ & $x$ & $x$ & $x$ & $x$ & \multirow{4}{*}[40,57]{} \\
\hline NFC & $x$ & $x$ & $\sqrt{ }$ & $x$ & $\mathrm{X}$ & $x$ & \multirow{3}{*}{ [41] } & Raspberry Pi & $x$ & $x$ & $x$ & $\sqrt{ }$ & $x$ & $x$ & \\
\hline EC-GSM-IoT & $\mathrm{x}$ & $x$ & $\sqrt{ }$ & $x$ & $\mathrm{x}$ & $x$ & & Gadgeteer & $\mathrm{x}$ & $x$ & $x$ & $\sqrt{ }$ & $x$ & $x$ & \\
\hline eMTC & $\mathrm{x}$ & $\mathrm{x}$ & $\sqrt{ }$ & $\mathrm{x}$ & $\mathrm{x}$ & $x$ & & Smart Phones & $x$ & $\mathrm{x}$ & $\mathrm{x}$ & $\sqrt{ }$ & $\mathrm{x}$ & $x$ & \\
\hline TinyOS & $x$ & $x$ & $\mathrm{x}$ & $\sqrt{ }$ & $x$ & $x$ & \multirow{2}{*}{ [40] } & Zig Bee & $x$ & $x$ & $\sqrt{ }$ & $\mathrm{x}$ & $x$ & $\mathrm{x}$ & \multirow{18}{*}[28,41,43,59]{} \\
\hline ContikiOS & $\mathrm{x}$ & $\mathrm{x}$ & $x$ & $\sqrt{ }$ & $\mathrm{x}$ & $x$ & & 6LoWPAN & $x$ & $x$ & $\sqrt{ }$ & $\mathrm{x}$ & $\mathrm{x}$ & $x$ & \\
\hline EPC & $\sqrt{ }$ & $x$ & $x$ & $x$ & $x$ & $x$ & \multirow{4}{*}[41,57]{} & T-GvoZB & $x$ & $x$ & $\sqrt{ }$ & $x$ & $x$ & $x$ & \\
\hline ubiquitous Codes & $\checkmark$ & $x$ & $\mathrm{x}$ & $x$ & $\mathrm{X}$ & $\mathrm{x}$ & & SigFox & $x$ & $x$ & $\sqrt{ }$ & $\mathrm{x}$ & $x$ & $\mathrm{x}$ & \\
\hline Ipv4 & $\sqrt{ }$ & $\mathrm{x}$ & $\mathrm{x}$ & $x$ & $x$ & $\mathrm{X}$ & & $2 \mathrm{G}$ & $x$ & $x$ & $\sqrt{ }$ & $\mathrm{x}$ & $\mathrm{x}$ & $x$ & \\
\hline Ipv6 & $\sqrt{ }$ & $\mathrm{x}$ & $\mathrm{x}$ & $x$ & $\mathrm{x}$ & $x$ & & $3 \mathrm{G} \& 4 \mathrm{G}$ & $x$ & $\mathrm{x}$ & $\sqrt{ }$ & $\mathrm{x}$ & $\mathrm{x}$ & $x$ & \\
\hline LTE-M1 & $x$ & $x$ & $\sqrt{ }$ & $x$ & $\mathrm{X}$ & $\mathrm{x}$ & \multirow{4}{*}{ [59] } & 5G & $x$ & $x$ & $\sqrt{ }$ & $\mathrm{x}$ & $x$ & $\mathrm{x}$ & \\
\hline UwB & $x$ & $x$ & $\sqrt{ }$ & $x$ & $x$ & $x$ & & LoRaWAN & $x$ & $x$ & $\sqrt{ }$ & $\mathrm{x}$ & $x$ & $\mathrm{x}$ & \\
\hline BLE & $x$ & $x$ & $\sqrt{ }$ & $x$ & $x$ & $x$ & & Ingenu & $x$ & $x$ & $\sqrt{ }$ & $x$ & $x$ & $x$ & \\
\hline WiFi-direct & $x$ & $\hat{x}$ & $\sqrt{ }$ & $x$ & $\hat{x}$ & $\hat{x}$ & & NB-IoT & $\hat{x}$ & $\hat{x}$ & $\sqrt{ }$ & $\hat{x}$ & $x$ & $\hat{x}$ & \\
\hline LiteOS & $x$ & $x$ & $x$ & $\sqrt{ }$ & $x$ & $x$ & \multirow[b]{2}{*}[40,59]{} & Collaborative-Aware & $x$ & $x$ & $\sqrt{ }$ & $x$ & $x$ & $x$ & \\
\hline L-Cat & $\mathrm{x}$ & $\mathrm{x}$ & $\sqrt{ }$ & $\mathrm{x}$ & $\mathrm{x}$ & $x$ & & Embedded sensors & $x$ & $\mathrm{x}$ & $\sqrt{ }$ & $\mathrm{x}$ & $\mathrm{x}$ & $x$ & \\
\hline RiotOS & $x$ & $x$ & $x$ & $\sqrt{ }$ & $x$ & $x$ & \multirow{6}{*}[40,57]{} & Bar codes & $x$ & $x$ & $\sqrt{ }$ & $\mathrm{x}$ & $x$ & $x$ & \\
\hline Android & $x$ & $x$ & $x$ & 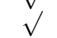 & $x$ & $x$ & & OWL & $x$ & $x$ & $\mathrm{x}$ & $x$ & $x$ & $\sqrt{ }$ & \\
\hline Nimbits-Cloud & $x$ & $x$ & $x$ & 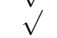 & $x$ & $x$ & & EXI & $x$ & $x$ & $x$ & $\mathrm{x}$ & $x$ & $\sqrt{ }$ & \\
\hline Hadoop-Cloud & $x$ & $\mathrm{x}$ & $\mathrm{x}$ & v & $\mathrm{x}$ & $\mathrm{x}$ & & Info. Aggr & $x$ & $\mathrm{x}$ & $\sqrt{ }$ & $\mathrm{x}$ & $\mathrm{x}$ & $\mathrm{x}$ & \\
\hline SmartThings & $x$ & $\mathrm{x}$ & $\mathrm{x}$ & 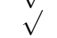 & $x$ & $x$ & & Ubiquitous & $x$ & $x$ & $\mathrm{x}$ & $\mathrm{x}$ & $x$ & $x$ & \\
\hline RDF & $\mathrm{x}$ & $x$ & $x$ & $\mathrm{x}$ & $\mathrm{x}$ & $\sqrt{ }$ & & Actuators & $\mathrm{x}$ & $x$ & $x$ & $\mathrm{x}$ & $\mathrm{x}$ & $x$ & \\
\hline
\end{tabular}

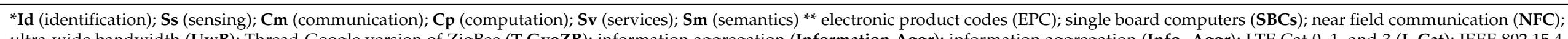

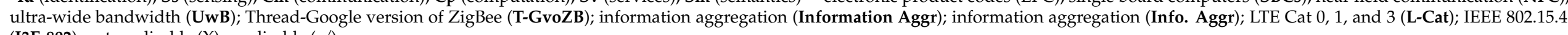
(I3E-802); not applicable $(X)$; applicable $(\sqrt{ })$. 


\subsection{IoT's Main Technologies}

Sensors and actuators (S\&As) are IoTs that need the enabling technologies presented earlier. These S\&As form the fabric of the IoT paradigm. Currently, there a different types of sensors as shown in Table 4. The list is still growing, and these S\&As are of different make and brand. The choice of which one to use is left to the discretion of users [20]. This presentation - to the best of the authors' knowledge and finding - is meant to guide stakeholders when deciding which sensor to design IoT solution with. As a paradigm, IoT aid communication between "things" and the role of sensors and actuators are pivotal to this. While sensors create information from events and happenings by sensing, actuators use such information to take action [60]. The sensing ability of IoT devices (sensors) comes from its ability to respond to stimuli and produce data, which are computable for inferential and predictive gains [61,62]. In LsM to have a never tiring eyes on ground is a great asset. Additionally, when there are ears to hear "things" and noses to smell as well then farmers can go to "sleep" and rest assured that surveillance, monitoring, and the perception (sensing) of salient occurrences that are difficult for humans are taken care of. This is where the use of S\&As comes in. IoT devices are designed to be deployed to take over the manual reading and clocking of observed happenings in the farm. Without the fear of fatigue, these tasks will be performed round the clock and nonstop. There are as many actuators, as there are sensors as shown in Table 4 .

Actuators are made for sundry purposes with appropriate delineations to respond to sensors, thereby accomplishing specific purposes. They complement sensors and essentially serve as the force that drives mechatronic systems (see Figure 4). While sensors transform electrical impulses that are construed easily and read, actuators sense (or receive) the readings as commands and actuate them into physical actions [63]. For example, a sensing unit can send data to a controller using a set of parameters. With this the controller-actuator makes necessary decisions using a control algorithm. The outcome of this is a command that actuate units to respond. In LsM, standard animal body parameters can be used to collect data through implanted biosensors, and such data will be sent to a controller that is within a central system for analysis. Multifunctional sensors also exist. They have optical and sound capabilities that can serve similar purposes as biosensors. Animal facial, body expression, and voice can be captured as data with S\&As such as biosensors. Data always make the difference, and with appropriate algorithms, billions of predictions can be made from which informed decisions are taken. Recent advances in mechatronics now make it possible to use robots to perform the administration of drugs and other sundry activities to benefit farmers due to data availability [64-66]. Contextually, LsM is a value-based scheme going by the traditional method of livestock practice of animal monitoring by which signs of injury or disease infection are identified. It is known that when an animal is ill, it is difficult to place a value on it. The value of an animal is a function of its health in relation to that of other healthy animals in a herd. Robots retrofitted with IoT can be valuable assert that uses available data to monitor the health of animals better than humans, since they are never fatigued and they can help overcome the pressure of labour shortages. This level of value addition and intelligence is some of the many supporting roles IoT systems will offer in LsM $[67,68]$. 


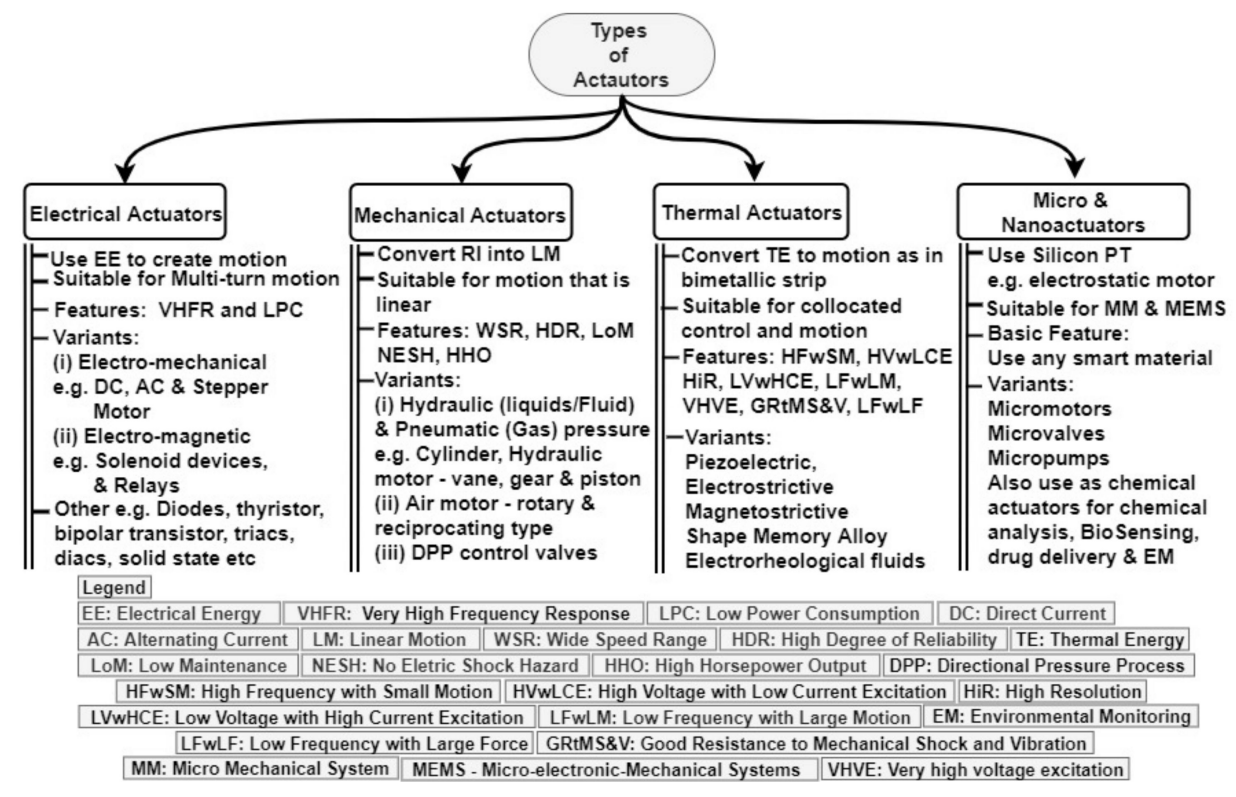

Figure 4. Different types of actuators.

Table 4. Types of sensors.

\begin{tabular}{|c|c|c|c|c|}
\hline Sensor Types & $\begin{array}{l}\text { Description/ } \\
\text { Uses }\end{array}$ & Make of Sensors & $\begin{array}{c}\text { Proprietary } \\
\text { Example }\end{array}$ & Cit. \\
\hline $\begin{array}{l}\text { Presence-position } \\
\text { proximity sensors }\end{array}$ & $\begin{array}{l}\text { Measure the presence, } \\
\text { position, and proximity } \\
\text { of objects }\end{array}$ & $\begin{array}{l}\text { Made of inclinometer, potentiometer, } \\
\text { and proximity sensors }\end{array}$ & $\begin{array}{l}\text { GPRS, GPS, and } \\
\text { transponders }\end{array}$ & {$[61,69-72]$} \\
\hline $\begin{array}{c}\text { Motion-occupancy } \\
\text { displacement } \\
\text { —velocity and acceleration } \\
\text { sensors }\end{array}$ & $\begin{array}{l}\text { Measures movement, speed, } \\
\text { wind, water direction, weight, } \\
\text { period, and pressure using } \\
\text { barometric means, } \\
\text { precipitation, motion } \\
\text { detection, and surveillance }\end{array}$ & $\begin{array}{l}\text { Made of radio detection and ranging } \\
\text { capacity-to sense skin temperature } \\
\text { and for microwave and ultrasonic } \\
\text { sensing; tomographic motion } \\
\text { detector-sensitive to disturbances; } \\
\text { video camera software for } \\
\text { photo-detection } \\
\text { and infrared lighting, and use as } \\
\text { accelerometers and gyroscope; } \\
\text { digital vernier calipers-senses size } \\
\text { of cracks }\end{array}$ & $\begin{array}{l}\text { Altimeter, sport sensors, } \\
\text { wearable sensors }\end{array}$ & {$[67,73-75]$} \\
\hline $\begin{array}{l}\text { Pressure, force, flow, and } \\
\text { load-strain torque sensors }\end{array}$ & $\begin{array}{l}\text { Measure pressure, volume per } \\
\text { time (i.e., mass flow or rate, } \\
\text { i.e., flow of velocity of fluid } \\
\text { and force }\end{array}$ & $\begin{array}{l}\text { Made of barometer, bourdon gauge, } \\
\text { piezometer-for pressure sensing; } \\
\text { viscometer, force gauge, tactile } \\
\text { sensor for touch sensing = for force; } \\
\text { flow sensor is made of an } \\
\text { anemometer, water, and mass meter }\end{array}$ & $\begin{array}{l}\text { Rotameters flow sensor, } \\
\text { spring and piston flow } \\
\text { meters, mass gas flow } \\
\text { meters, ultrasonic flow } \\
\text { meters, turbine flow } \\
\text { transmitter, etc. }\end{array}$ & {$[62,76,77]$} \\
\hline $\begin{array}{l}\text { Acoustic-vibration and } \\
\text { sound sensors }\end{array}$ & $\begin{array}{l}\text { Measure sound levels and } \\
\text { convert that information into } \\
\text { digital/analogue data signals }\end{array}$ & $\mathrm{N} / \mathrm{A}$ & $\begin{array}{l}\text { NDI sensor, ultrasonic PD } \\
\text { sensors, UHF sensors, } \\
\text { geophone, microphone, } \\
\text { hydrophone }\end{array}$ & {$[62,78,79]$} \\
\hline $\begin{array}{l}\text { Humidity, moisture, and } \\
\text { temperature sensors }\end{array}$ & $\begin{array}{l}\text { Measure levels of relative } \\
\text { humidity, cold, or heat; } \\
\text { identify physical contact or } \\
\text { lack of contact using } \\
\text { convection, radiation, and } \\
\text { presence of water, air, and } \\
\text { toxic gases }\end{array}$ & $\begin{array}{l}\text { Made of organic polymer (e.g., } \\
\text { polyethylene, polyamide resin, or } \\
\text { metal oxide); wet and dry bulb } \\
\text { psychro-meter and a mechanical } \\
\text { hygrometer; EMC—a forward } \\
\text { meson spectrometer, thermistors, } \\
\text { and RTDs }\end{array}$ & $\begin{array}{l}\text { Humistor, hygrometer, } \\
\text { moisture sensor, } \\
\text { calorimeter, thermometer, } \\
\text { temperature gauge }\end{array}$ & {$[62,67,80,81]$} \\
\hline $\begin{array}{l}\text { Optical (i.e., light), machine } \\
\text { vision, ambient light } \\
\text { sensors }\end{array}$ & $\begin{array}{l}\text { Measure and detect the } \\
\text { presence of light (i.e., invisible } \\
\text { or visible), monitor real-time } \\
\text { temperature, measure and } \\
\text { detect } \mathrm{pH} \text {, refractive indices } \\
\text { voltage humidity, antigens, } \\
\text { chemical species }\end{array}$ & $\begin{array}{l}\text { Made of photoelectric device of } \\
\text { either photo-emissive or voltaic } \\
\text { materials, photo-resistors or } \\
\text { conductors; LED drivers, infrared, } \\
\text { LDR from cadmium sulphide, etc., } \\
\text { depending on the area of application }\end{array}$ & $\begin{array}{l}\text { Real-time temperature } \\
\text { monitoring (infrared), } \\
\text { ultraviolet light, } \\
\text { LMR-based optical sensors, } \\
\text { and many others based on } \\
\text { area of application }\end{array}$ & {$[62,67,82-84]$} \\
\hline Radiation sensors & $\begin{array}{c}\text { Measure and detect radiation } \\
\text { in an environment based on } \\
\text { ionization and/or scintillating } \\
\text { detection }\end{array}$ & $\begin{array}{l}\text { Made of thermoluminescent } \\
\text { dosimeter, semiconductor, wilson } \\
\text { cloud chamber, and carbon } \\
\text { nanotubes }\end{array}$ & $\begin{array}{l}\text { Scintillator, Geiger-Müller } \\
\text { counter, neutron detector }\end{array}$ & {$[62,67]$} \\
\hline
\end{tabular}

Global positioning system (GPS); general packet radio service (GPRS); northern digital incorporated (NDI); partial discharge (PD); ultra-high frequency (UHF); electromagnetic calorimeter (EMC);resistance temperature detectors (RTDs); light-dependent resistor (LDR); lossy-mode resonance (LMR); Citation (Cit); not available (N/A). 


\subsection{IoT's Technicalities and the Livestock Context}

The use of IoT to deliver services is technically reliant on what happens at the middleware layer of the IoTA. This pertains to how the perception, networking and application, and processing layers are managed. In recent IoTA, the initial layers have been extended with the middleware strengthened to serve as the service management layer. In the new proposals, the networking and application layers were separated to make the IoTA a five (5)-layered architecture (see Figure 2b) [21,58]. This confers individual tasks on each layer of the IoTA with unique technicalities. In the perception layer, sensing is done using variable physical quantities (VPQ) to sense sound, humidity, light intensity, and temperature, etc. In the context of managing livestock with smart technologies, this VPQ will be resourceful in making it possible to use IoTT on a large scale in animal farms. Technically, IoT vis-à-vis its functionalities will leverage these VPQs to offer the opportunity for IoTT to play its monitoring role. This implies that on a massive scale animal growth, wellbeing, and welfare can be monitored unlike humans would do on a small scale. The advantage in this is the fact that it is possible to codify VPQ to use IoT to collect data, pre-process, and compute them for appropriate action. The collection of data takes place in the perception layer but are transmitted/routed through the Internet in the network layer. Some of the Internet technologies to contemplate in this regard are: WIFI, 5G, Zigbee, 3G/LTE, and Lora.

The application layer is where the ultimate goal of IoT-which is service delivery-is met [58]. In the management/middleware layer (MML), a lot of computation is carried out as against fewer ones in the perception layer. Every processing in these layers are coordinated in the processing layer. Interactions in these layers often use a real-time active database [21,42]. The computational activities in the MML is borne out of the use of open lightweight and secure IoT middleware operations [42]. The IoT's MML provides three frameworks to allow its workability, namely, the service-oriented architecture (SoA), cloudbased solution (C-bS), and the actor-based framework (A-bF). While the SoA is responsible for regulating the deployment and addition of any category of IoTT as a service and as the need arises, the C-bs controls the number and type of IoTT that are deployable per time. The A-bF is responsible to provide an open plug and play standardization platform for sensors and actuators to operate using VPQs. All of these will provide an application plane to handle routine (utility) services. In LsM, there are routines. IoTT being a routine-oriented technology makes it fit to handle routine tasks. With VPQs, IoTT can exert control and manage repetitive tasks and save labour cost [85]. For example, after monitoring through sensing, IoT sensors will alert farmers when a VPQ falls out of the normal range at real-time or near real-time with individualized information about individual animals. As such, the cost of physically, laboriously, and repetitively checking the vitals of each animals will be reduced.

\section{Review Method}

To be consistent with what obtains in the literature $[2,86,87]$ and reach the aim of this review, PRISMA — the methodology for reporting preferred items for systematic reviewswas adopted to provide the framework to support the systematic literature review. The workflow of the framework is shown in Figure 5.

It simplified the systematic review procedure using PRISMA that guided the concerted research strategy applied starting from June 2019 through to August of the same year, with a search made to SCOPUS database to source for and identify the relevant articles for this work. Other indexing databases such as EBSCO, DBLP, and Web of Science (WoS) were also searched for wider coverage (see Figure 5). These databases also index highly rated journals and catalogues that include that of ACM, IEEE, etc. [2]. 


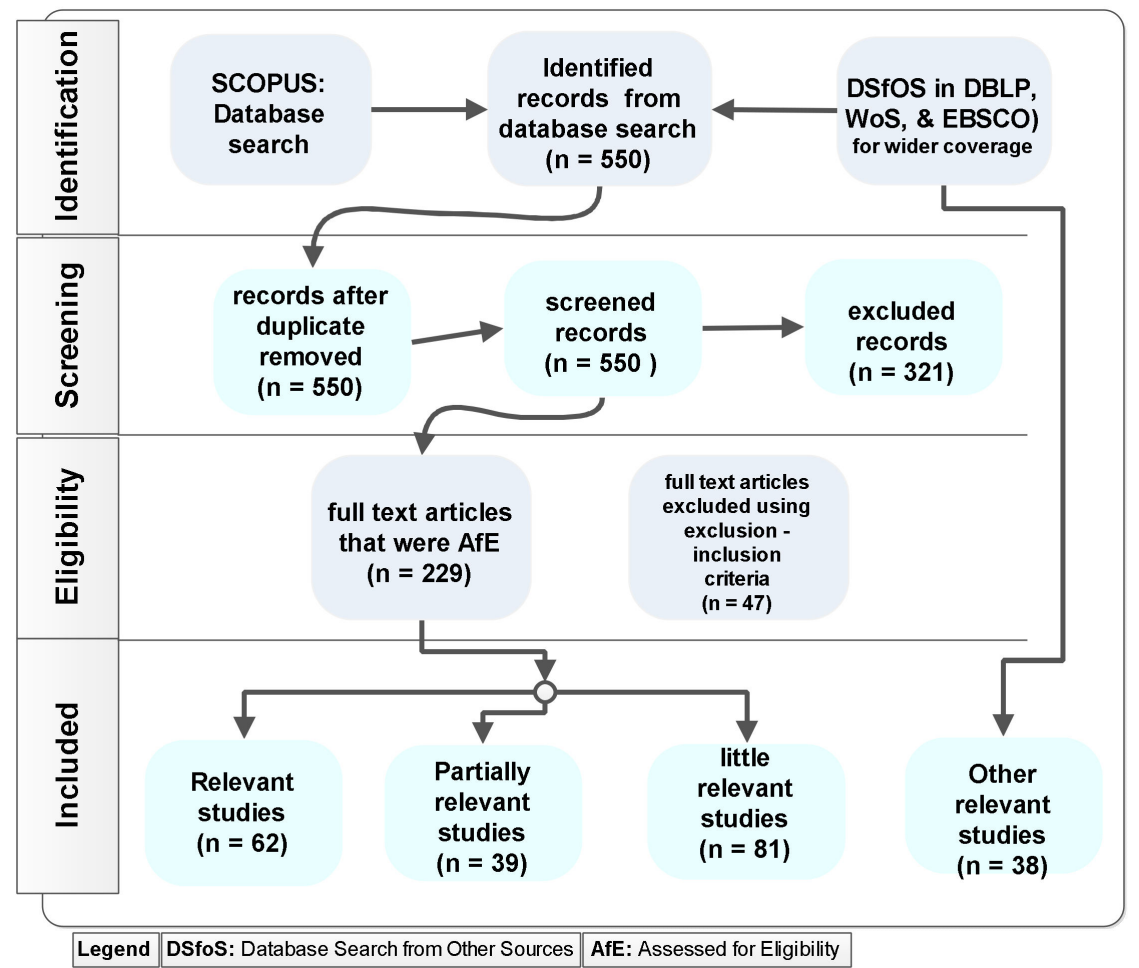

Figure 5. The systematic review procedure using PRISMA.

Using the PRISMA framework, in the identification stage, articles were manually screened, and then duplicate record(s) (i.e., articles) were identified and removed. Titles, abstracts, and keywords in the articles and the objective to use IoT for problem solving guided the screening and use of eligibility criteria. In the eligibility stage the inclusionexclusion criteria were applied as prescribed in [2]. In the final stage, qualitative synthesis of included studies was carried out. These articles were further categorized into relevant studies (62), partially relevant studies (39), little relevant studies (81), and from other relevant studies (43). This made it possible to have a detailed study carried out on the articles in the qualitative synthesis activity in the "include and final stage".

The search criteria used keywords such as "(IoT OR Internet of Things)" and "(Livestock OR Animals)". Keywords that include sensors, actuators, IoT ecosystem and architecture, the role of IoT, etc., were also leveraged in the search process. Following the practice in [86] and [2] to improve the search process, effort was made to ensure that the keywords were present in the article abstract, title, highlights, or article keywords, and the articles that were considered were published in English. Additionally, articles that are related to core agricultural thematic were excluded. By relevance, the articles that pertain to the subject under consideration were grouped together as relevant, since they directly focus on the role/application of IoTT in the livestock context. Articles from which this objective could be inferred were also included in this category. Whereas articles that were moderately relevant to certain IoT technologies and enabling technologies in relation to LsM were included to the category of partially relevant, the ones whose topic was off the objective of this paper and/or lacked originality as well as the rigor of science due to lack of peer-review were categorized as little relevance [86]. Much progress was made in this process, when a more concerted search was repeated between January and March of 2020 and in the month of December 2020. At the final stage, the analysis resulted in 182 articles. When added to the articles that are relevant from other sources (38), a total of 220 articles were considered suitable and included as the sample for the state-of-the-art review. 


\section{IoT and Its Cyber Physical System Perspective}

This section presents the cyber physical systems (CPS) perspective of IoT. As earlier presented, IoT and its emerging technologies have been interrogated to ascertain what IoT require to deal with CPS concerns among others. The CPS perspective of IoT presents the lens to understand the scale at which IoT would operate to initiate analytics using data to drive growth in LsM. This scale of operation in the role IoT will play to manage livestock going by the conception of CPS will amount to introducing the industry 4.0 scale of resource management. This refers to large-scale production by digitalization that entails the use of advance and emerging technologies such as big data, IoT, and cloud computing. The connection of physical object with the digital world under the CPS perspective will be the burden of IoT, while big data techniques will take care of the data that will result from the connection. This implies that production by digitalization and its management would be run and managed relying on algorithms. This will unlock the meaning in data to set off analyses, of which the result will be useful to manage and improve processes within the farm. This section is significant in that IoT's synergistic role with machine learning (ML), and AI regarding data analysis is unveiled with algorithms highlighted as what will drive the production of value and utility from data.

\subsection{CPS Perspective of IoT in Livestock Management vis-à-vis Big Data}

In the CPS architecture [88-90], IoT is part of the physical system of the CPS. In this subset, there also exists the Internet of knowledge and content (IoKC), which specifically entails data that are translatable to knowledge as useful information. The relation that exists within the context of the CPS is that of interactions based on joint collaboration and action of IoT and IoKC. The cyber system part of the CPS model offers the service orientation that allows big data to result from the sensing potentials of the IoT [88]. CPS entails control (i.e., management) through computation and communication. While computation will be handled by algorithms as reveal in sub-section on algorithms, communication will be made via the Internet that will offer the data infrastructure to operate in.

IoT from CPS perspective is a transformative technology to manage systems that are interconnected. This is uniquely important in LsM where the context of operation falls with the system interconnectedness conception, where physical assets possess the potential for computing. As highlighted in [10], when CPS is integrated with production and services, its management would result in the industry 4.0 scale. Based on the concept of smart farming that allows the extensive adoption of IoT from the CPS perspective, generated data from smart devices from different user applications will be easily harnessed. In [89], a multi-layer architecture that accommodates the possibility of harvesting data from different multi-cloud scenarios in the context of smart farming was presented. There is a future in this that encourages the industry 4.0 scale of the digital management of livestock farming. LsM that countenances heavy automation, real-time data collection capability, and machine learning with interconnectivity can be operated in synergy to physical production and operations using smart digital technology [91]. Table 5 presents information about the potential of IoTs to deliver big data through the lens of CPS as an advantage to LsM. While the table also includes information about CPS's potential limitation, Table 6 presents a comparative evaluation of current studies in the field of CPS to the benefit of IoT for LsM. The analysis and comparison presented in Tables 5 and 6 follow best practice in the literature e.g., in [39]. 
Table 5. An inductive analysis of IoT as a CPS to deliver big data.

\begin{tabular}{|c|c|c|}
\hline Advantages & Limitations & Cit. \\
\hline $\begin{array}{l}\text { - Identified big data business-oriented model (BDBoM) } \\
\text { that is effective in associating data-driven technologies of } \\
\text { big data scale. The conception uses MLT and algorithms to } \\
\text { support real-time clarifications analytically to improve } \\
\text { sustainable production. }\end{array}$ & $\begin{array}{l}\text { - Sustainable production rely on inputs that } \\
\text { are trustworthy, which still need improvement in } \\
\text { the BDBoM. }\end{array}$ & [92] \\
\hline $\begin{array}{l}\text { - Motivated by the problem of scalability CPS mechanism } \\
\text { for decision making to meet data storage and processing } \\
\text { requirements. The framework allows the use of low-cost } \\
\text { orchestrators that demonstrated satisfactory control in } \\
\text { micro-grid environment. }\end{array}$ & $\begin{array}{l}\text { - Still requires the removal of operational } \\
\text { constraints from target app domain that imposes } \\
\text { sensors to continuously monitor manifold } \\
\text { scenario of operations that hinders optimal } \\
\text { configuration }\end{array}$ & [93] \\
\hline $\begin{array}{l}\text { - The paper presented a multi-layer architecture that } \\
\text { captures numerous entities that can handle real-time use } \\
\text { cases based on support from the cloud and edge } \\
\text { environment. }\end{array}$ & $\begin{array}{l}\text { - Though there was a real test bed for the } \\
\text { architecture, cyber-attacks on CPS may still } \\
\text { easily unleashed. } \\
\text { This attacks are easy due to leakages of } \\
\text { information through unauthorised access or } \\
\text { insider intruders that are potential threats. }\end{array}$ & [89] \\
\hline
\end{tabular}

- Identified a feedback loop that CPS feedback loops were identified as effective to integrate both the physical and the cyber subsets of CPS since how they are integrated affects their operation.

- $\quad$ This achieved effect data analysis, and both physical and data-driven, and data fusion capacity.

- In turn, knowledge discovery is easily enhanced even from heterogeneous sources of data, which is of a high-dimensional data scale, thus, scalability is ensured.

- $\quad$ Overfitting still remains a troubling challenge.

- Introducing computational intelligence enforces the use of "black box" algorithmic techniques, of which inner workings and behaviour are difficult to understand and explain.

- The study characterised and presented a reference architecture to implement a big-data-oriented CPS.

- $\quad$ The architecture was presented as a 9-tuple that includes that capture the social CPS that fosters IoKC.

- Security and the challenge of heterogeneity of "Things" remain major issues. 
Table 6. Indicative comparison evaluation of current studies on CPS.

\begin{tabular}{|c|c|c|c|}
\hline Features & Description & Limitation & Remarks/Cit. \\
\hline $\begin{array}{l}\text { - A novel decision-making } \\
\text { technique (DMT) that operates } \\
\text { online and optimally configures } \\
\text { CPS is proposed. } \\
\text { It showed low storage and } \\
\text { computational difficulties in a } \\
\text { ventilation, heating, and air } \\
\text { conditioning (VHAC) context } \\
\text { (i.e., in a micro-grid setting). }\end{array}$ & $\begin{array}{l}\text { The DMT uses a multiple-choice } \\
\text { knapsack and linear regression } \\
\text { algorithms that accurately } \\
\text { estimate VHAC's energy usage. } \\
\text { It is capable of balancing out } \\
\text { energy consumption with the } \\
\text { energy budget intact. }\end{array}$ & $\begin{array}{l}\text { - Additional data are not able to } \\
\text { improve the proposed model accuracy, } \\
\text { since it imposes constraints to the } \\
\text { procedure of refinement. }\end{array}$ & $\begin{array}{l}\text { - Showed the existence of } \\
\text { prototyping framework to implement } \\
\text { and customize CPS in domains (such } \\
\text { as livestock farming) where decision } \\
\text { making is critical and on the spur of } \\
\text { the moment. [95] }\end{array}$ \\
\hline $\begin{array}{l}\text { Proposed an architecture with } \\
\text { multi-layers that reflect diverse } \\
\text { user applications that fits into } \\
\text { various layers for a distributed } \\
\text { cyber physical environment } \\
\text { such as the smart farm } \\
\text { ecosystem. } \\
\text { Security and other privacy } \\
\text { concerns with different attack } \\
\text { scenarios were highlighted. }\end{array}$ & $\begin{array}{l}\text { - The architecture adapts CPS and } \\
\text { IoT design to utilize the infinite } \\
\text { abilities provided by edge and cloud } \\
\text { services to harness data from smart } \\
\text { devices and handle numerous user } \\
\text { applications at its layers. }\end{array}$ & $\begin{array}{l}\text { - Regarding implementation } \\
\text { assumptions, no information is } \\
\text { available. } \\
\text { A framework is needed to } \\
\text { guide the elicitation of } \\
\text { implementation requirements } \\
\text { within the context of } \\
\text { softwarization to protect } \\
\text { sensitive data. }\end{array}$ & $\begin{array}{l}\text { - Show that in distributed and } \\
\text { dynamic cyber physical environment } \\
\text { where IoT devices are adopted across } \\
\text { several domains, privacy and security } \\
\text { issues are inevitable. [96] }\end{array}$ \\
\hline $\begin{array}{l}\text { - The study highlighted the CPS } \\
\text { that is driven by big data analytics, } \\
\text { deep learning-assisted smart planning } \\
\text { process, and decision-making } \\
\text { cognitive algorithms as a digital } \\
\text { technology. }\end{array}$ & $\begin{array}{l}\text { - IoT sensing and computing } \\
\text { networks in synergy with AIbDMA } \\
\text { and blockchain (to aid big data } \\
\text { innovations) were shown as capable } \\
\text { of sustaining production at large scale. }\end{array}$ & $\begin{array}{l}\text { - No framework was suggested } \\
\text { for possible implementation. } \\
\text { No implementation details or } \\
\text { assumption were provided. }\end{array}$ & $\begin{array}{l}\text { - Show that digital technologies can } \\
\text { be used with state-of-the-art } \\
\text { algorithm to assist during } \\
\text { de-carbonization process. [92] }\end{array}$ \\
\hline $\begin{array}{l}\text { - The research work introduced IoT } \\
\text { as capable of unlocking new } \\
\text { capabilities to enhance performance } \\
\text { by organizing internal logistics. A } \\
\text { design perspective was adopted for } \\
\text { IoT-driven analytics within CPS } \\
\text { context and approach. }\end{array}$ & $\begin{array}{l}\text { - The CPS demonstrator offers } \\
\text { (and guide) data generation } \\
\text { with performance estimation } \\
\text { and appropriate visualization. } \\
\text { As an IoT-driven CPS, its } \\
\text { performance analytic } \\
\text { monitoring can benefit the } \\
\text { upgrade of legacy systems. }\end{array}$ & $\begin{array}{l}\text { - There is the absence of efficient } \\
\text { synchronization mechanism to } \\
\text { exploit production assets and } \\
\text { spur the uptake of technological } \\
\text { upgrades. } \\
\text { Data complexity and } \\
\text { interoperability concerns } \\
\text { require much attention. }\end{array}$ & $\begin{array}{l}\text { - Validated the fact that } \\
\text { performance monitoring is } \\
\text { visualize-able and CPS tuned to } \\
\text { support operational performance and } \\
\text { sustainability. [97] }\end{array}$ \\
\hline
\end{tabular}

- $\quad$ An IoPT that integrates reliable machine learning and data analytics methods to sure-up the resilience of CPS against cyber-attacks.

- $\quad$ The concept was validated using the testbed of CPS under randomly selected cyber-attack scenarios to satisfy the proof of concept.
- $\quad$ The IoPT is a hybrid CPS that uses state-of-the-art IoT, data science, IIS, and edge analytics.

- Its architecture embeds AI-dA and supports less load of computation that is free of latency in the cloud.
- The feasibility of exploring another anomaly detection technique is encourage to curb adversarial ML attacks.
- Demonstrate the fact that CPS can become or be made resilient against a variety of cyber-attack scenarios. [98]
- A framework capturing the implementation of the CPASs. It intelligently integrates CPS that leverages IoT, big data, and $\mathrm{CC}$ with agricultural systems.
- CPAS was highlighted to boost productivity with potential in diary and meat production.
- More implementation details to actualize the CPASs in real-time agric-systems are still needed.
- Shows that the CPS conception using industry 4.0 technologies can increase sustainable productivity in the agric-sector. [99]
- $\quad$ Presents a typology that captures different digital twins (DTw) as CPS in conceptual framework.

- It incorporates a control model based on IoT architecture with proof of concept validation.
- The framework deploys DTw and allows reasoning and learning from real-time data and other data sources to dynamically recalibrate and make improved decision.
- $\quad$ No discussion regarding privacy concerns were presented.

- $\quad$ The framework was built on analysis of literature and is still developing.
- The framework is particularly useful since the DTw is good at removing constraints that impose physical proximity. [100]

Citation (Cit.); cyber physical systems (CPS); artificial-intelligence-based decision-making algorithms (AIbDMA); Internet of Predictable Things (IoPT); AI-driven algorithms (AI-dA); machine learning (ML); cyber physical agricultural systems (CPASs); cloud computing (CC); intelligent industrial systems (IIS).

\subsection{Algorithmic Perspective of IoT and CPS in Livestock Management}

Livestock farmers are saddled with intricate responsibilities. Sometimes, this includes working out the optimum method(s) to apply to several tasks, most of which are repetitive. So, the onus is on livestock farmers to regularly make life saving and profit making 
decisions. This burden can easily be borne using a modern technique-algorithms in ML. IoTT can leverage them in a win-win situation. This CPS-IoTT perspective comes with a technical burden, for which algorithms exist. Algorithms would drive all forms of computation including data analytics to manage and implement modern business models in livestock production. Interestingly, the algorithmic support component of the IoT-based system is as essential as sensors and actuators and other IoTTs. For instance, algorithms, especially in the genre of ML, would be used by systems to interpret sundry activities. This means more objective information about individual animals will be easily provided to support farmers to make better choices about what works to sustain production. In livestock farming, there are two main costs: feed and the management of diseases aside from stocking rate as the basic driver of cost. These costs can be better managed using existing advanced technologies (e.g., AI and ML algorithms) when deployed to help farmers make sense out of costs data, about which insight would guide the decision to determine best feed rates, diet formulations, and disease treatment regimen, etc. [101].

In $[102,103]$, a wireless bioelectric system known as canine body-area network that uses low-power consumption was reported. This advanced technology uses ML algorithm to give feedback from sensors and actuators. With the right interpretation of such feedback, health and welfare issues can be resolved to better the life of livestock. Oestrus-a cycle of reproductive activity in animals-is an important moment such that when properly managed animal breeding becomes easy and successful. IoT-based systems can detect and observe this moment unobtrusively for several animals on a large scale simultaneously. There is also an anomaly detector that uses the support vector data description methods. In the literature [104], it was used to detect oestrus sound, thus affirming the possibility of the early detection of anomaly in oestrus using ML and sound data. In a typical livestock farm that adopts the IoT technicalities for LsM, there will be a large continuous inbound data stream that is characteristic of surveillance systems. Though the tasks of oestrus monitoring and management is repetitious and to be done discreetly, it is one example of the many repetitive tasks in a typical livestock farm [72]. With IoT-based systems, all of these tasks can be handled automatically. The detection and observation of oestrus moments can also be done unobtrusively for several animals on a large scale simultaneously using IoT as a CPS. Sound from animals such as coughs are gestures from which audio features can be extracted using supervised learning algorithms such as k-nearest neighbours, support vector machine, random forest, and bootstrap aggregations [105], etc., to monitor animal health far better than using manual approach. LsM needs an integrated environment with the potentials for intelligence to thrive, thus the need to introduce the CPS conception. This demands a cognitive automation process (CAP) that is computationally driven. Artificial intelligence (AI) algorithms suffice in this regard to intelligently process and analyse data to deliver critical improvement when CAP is in place. The confluence of AI algorithms and IoT can then play the support role of intelligent data processing (IDP) in LsM.

The role IoT will play regarding IDP is the provision of the means of identification, sensing, communication, computation, services, and semantics. These IoT features (i.e., elements) will be deployed to man distinct activities and tasks areas that befit them in LsM for management purposes. In Figure 6, some of the techniques (i.e., algorithms) to be delivered by AI are shown in the left had side of the diagram in Figure 6. These techniques are poised to support IoT's management role in LsM by providing the method for IDP. The fusion and integration shown in Figure 6 is meant to report the techniques (see left hand side (LHS) of the diagram in Figure 6) that will be relied on to support IoT in playing its role of identification, sensing, communication, etc., as shown in the right hand side (RHS) of the diagram in Figure 6. Much as the integration captured in Figure 6 may not be perfectly done, it, however, shows IoT roles vis-à-vis the intelligent job functions it will perform on behalf of livestock farmers on a daily basis. These intelligent job functions in summary include identification, sensing-by observation, semantic - giving the right interpretation, communication, etc. 


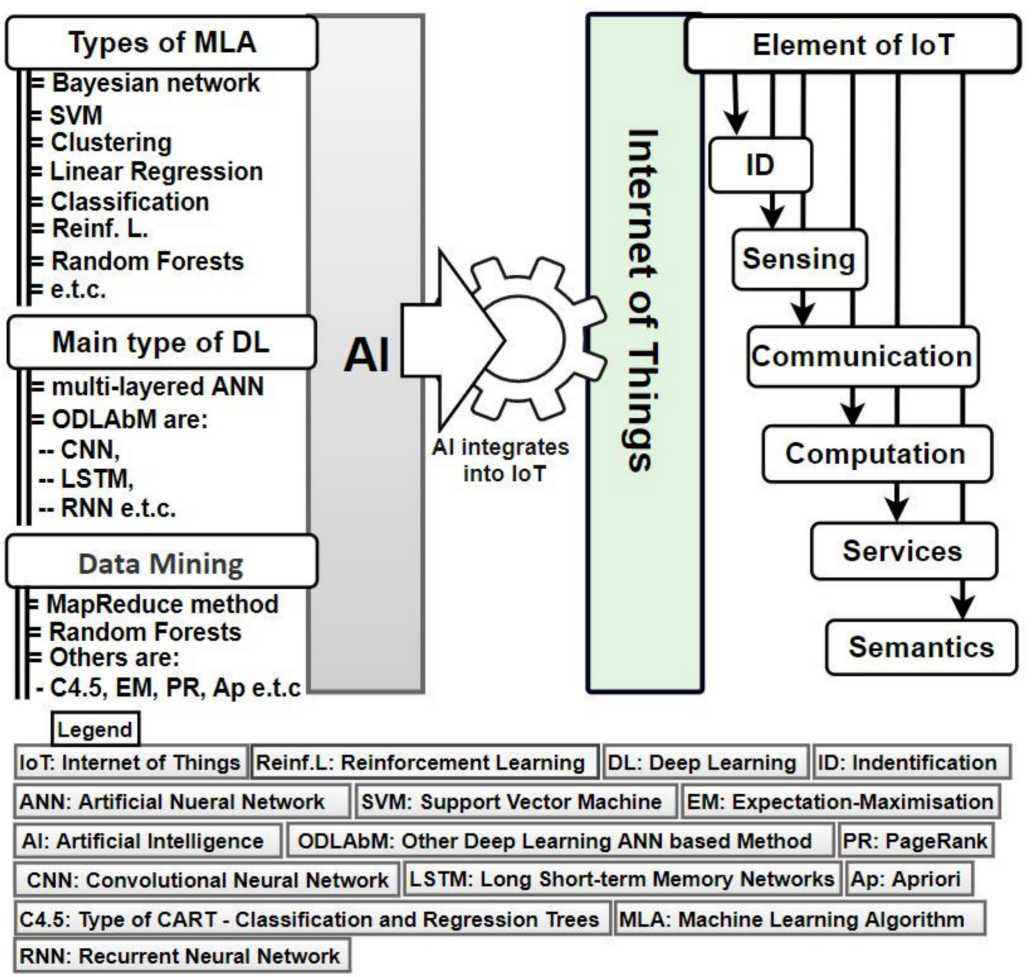

Figure 6. Showing IoT characteristics (i.e., role) that integrate AI by way of being helped to take the management responsibility in LsM.

IoT will perform this intelligent job function much better than livestock farmers, who need help with the management of scarce resources (especially that of land) as well as assess situations on the spot with prompt feedback. Anomaly detection and root cause analysis, which relates causal-effect relationships, and other recurrent tasks, etc., can also be handled digitally in LsM $[101,106]$. AI techniques fall mainly within the machine and deep learning and data mining domain (see LHS of the diagram in Figure 6). A confluence of these techniques is possible for either learning or mining with AI techniques irrespective of the domain of application. With brevity, machine learning is presented as follows to help enlighten readers on its knowledge and application in the next section.

\subsection{Machine Learning}

Machine learning (ML) algorithms are of several types such as artificial neural networks, clustering and classification, and regression methods. These ML techniques will be used to provide the system and device intelligence (SandDI) that is needed for IoT elements to demonstrate their capabilities as expected. In LsM, reliance on this SandDI can help resolve key environmental, production, and sundry other tricky challenges [6]. IoT devices will provide extensive data that will be analysed by advanced ML and AI algorithms to notify farmers after prediction should there be any abnormal situation [101]. With advanced ML and AI algorithms sensors and the data they provide, they can synergistically be relied on to provide solution in the context of livestock farming and management $[101,107]$. The support expected from ML methods (in the role of IoT in LsM) in real time is an abstract data representation of sensor observations $[107,108]$. Bayesian network is an example ML technique (MLT), which can support knowledge representation in domains such as LsM where uncertainty is rife. Using data from IoT devices, this technique like others will benefit LsM in that the knowledge to manage uncertainties will be realised from such data instead of wild guessing.

Based on the literature [101], a confluence of AI and ML algorithms will serve as a candidate technique to determine the status of animal health and wellbeing. Using the technique to mine and analyse data from IoT devices the paradigm of IoT will digitally 
be resourceful to play its management role without guesswork as was the case in the traditional method of livestock management. From the same data, which IoT devices will collect from sundry animal activities, augmented analytics can be deployed to monitor animal heart rate, location, blood pressure, temperature, etc., remotely. Without distorting the natural ecosystem and its environment, which is home to animals, advanced AI and ML techniques can be used to model and simulate possible scenarios to learn about weather and even provide guidance on the reduction in livestock contribution to greenhouse gas emissions, etc. [109]. The pattern recognition that is possible with AI and ML algorithms will yield an immediate prediction that will be useful within the context of LsM to weigh and estimate many options and then introduce economic balances accurately. With these advanced digital techniques, the livestock production line is poised to have a support mechanism that is guaranteed of procedure to manage the complexities and uncertainties faced by livestock farmers on a regular basis [110].

\section{IoT Application and Usefulness in Livestock Management}

LsM is all about resource management. Two important resources to manage livestock production line are (i) the quality of life (QoL) of livestock and (ii) the condition of the land where animals are farmed. Since the context of this work is livestock farming, the authors focus is on managing the QoL of livestock using IoT. Therefore, the management of biosecurity, animal welfare, and product quality control, animal recognition through identification is overarching. This is because these areas, which are to be managed regarding livestock, will play a critical role in ultimately determining the QoL of animals and in turn show their health and welfare status. Nevertheless, the discourse of biosecurity, animal welfare, and product quality control, etc., is delved into after the comparison in Table 7 that highlights the pros and cons of some relevant studies on the application of IoT in LsM following the practice in [39]. 
Table 7. IoT application in livestock management.

\begin{tabular}{|c|c|c|}
\hline Comment on Paper/Citation & Advantages & Disadvantages \\
\hline $\begin{array}{c}\text { Digital twins (DTws) can bring new levels of } \\
\text { farming that is sustainable at large scale of } \\
\text { productivity }[100,111]\end{array}$ & $\begin{array}{l}\text { The framework is conceptual but effective } \\
\text { in guiding the design and implementation of } \\
\text { DTw-based farm management system that } \\
\text { applies a typological approach to leverage } \\
\text { DTw to plan, control, monitor, and optimize } \\
\text { farm processes. }\end{array}$ & $\begin{array}{l}\text { - A detailed reference architecture for } \\
\text { modelling and realizing the DTw-based IS is } \\
\text { still absent. }\end{array}$ \\
\hline
\end{tabular}

- This framework is advantageous in that it provides sensor layer to use devices that are sensor-rich to collect contextual livestock-oriented data, and a layer for data processing to detect sick animals, animals on heat, etc.

The synergy of IoT and deep learning (DL) brings livestock monitoring to a resourceful level that supports the effective management of livestock digitally [112]

- Insightful information can, therefore, be sent to herd owners, and production cost is effectively managed.
- Although some level of implementation details were shown, privacy and security concerns were unattended to.
Acoustic monitoring technique based on DNN was shown to be better than the use of HMMT to monitor animal activity [113]
- Use of DNN for an efficient DL acoustic activity technique for modeling lossy compressed or uncompressed bee sounds.

- $\quad$ The architecture made provision for modification in the hidden DNN layers, which is the advantage over HMMT.
- Poor network response that hinders real-time transmission of audio signals and insufficient computing capability still need attention.
IoT-based LoRa network demonstrates field ready, rapidly deployable, and flexible capacity to monitor pastured livestock activity (i.e., behaviour) and location identification in expansive environments [96]
- This network removes the bottlenecks of networking access that facilitates regular animal contact.

- It provides realistic alternative to cellular or WiFi networking solutions that are available for extensive systems.

- Its potential to use machine-to-machine IoT protocol of connectivity makes it more viable to transfer data at wide range in several kilometers.
- There is need to build upon the tool to optimize the frequencies of reporting, improve power requirements, and refine accuracy and precision of classification potentials for (e.g., animal behaviour) so as to update the advancement of wearable IoT technologies for extensive system of production such as livestock farming.
- IoT framework allows the use of behavioural time-series data that are multivariate in nature. Neck-based mounted sensors are applied to collect the multivariate data.

Mating time are trackable to know optimal time of oestrus as a critical constituent of reproductive management in animals using IoT technologies [114]
- Its a cost-effective detection method that uses real-time ML-detection-based algorithms with low power consumption and wide coverage capacity.

- $\quad$ Patterns are extracted from oestrus data by exploiting DNN and LSTM to give oestrus alert.
- Although efficient for oestrus detection, the framework still needs extension to be fit to implement efficient monitoring digital systems to cater for (i.e., monitor) disease, health, and abortion, etc., in animals.
IoT-based digital technology was countenanced as resourceful in the assessment of QoL of livestock and by extension their on-farm welfare in real time for all animals [115].
- The framework identifies measures to adapt animal welfare assessment (AWA) to bridge existing knowledge gap to benefit the assessment of QoL of livestock.

- $\quad$ From AWA measures, digital representations are built to improve animal monitoring, and in ILF, this will be useful in address animal welfare challenges such as heat stress, pressure body/wounds, early weaning practices, lesion susceptibility, and tail biting behaviour, etc.
- $\quad$ Still impossible to observe individual animals using detached sensors to observe (several) animals per sensor.

- $\quad$ Some other identified knowledge gaps to be filled include the welfare monitoring of not only at the abattoir-a few studies have handled this, but during transport particularly inside large enclosed vehicle environment where observation is manually difficult for humans, etc. 


\subsection{Biosecurity}

In biosecurity, proactive measures are set up to prevent the outbreak of diseases. It also entails the control of disease outbreak when it occurs. When animals are sick, the human population will be at risk, since they can be infected and become sick when products from sick animals get into the market. With proactive measures important biosecurity steps are taken to prevent the outbreak of diseases. This measure includes the use of control to reduce disease transmission, while productivity parameters are maintained. The standard practice in biosecurity is to prevent the spread of endemic outbreaks by checkmating the causative agents [116]. Integrated structures must be in place to run different types of risk analysis on human-animal activities to identify causative agents so as to check zoonoses diseases before they are transferred from animals to humans or vice versa $[117,118]$.

Contextually, early responses to disease outbreaks through proactive collaborative approach would require risk assessment along with well-informed, coordinated, and prompt information sharing. Coming from this possibility, authorities using existing integrated structures that are interconnected can enforce robust control. IoT's integration with existing interconnected structures would make data and the possibility to monitor how members uphold protocols available. Response to zoonotic attacks is best when there are tailored surveillance strategies to adapt in the face of rapidly changing objectives during zoonotic disease activities $[117,118]$. At regional and country levels, coordinated efforts to put zoonosis at bay have been premised on existing parameters and protocols (P\&Ps). This list of P\&Ps can be endless. Moreover, more attention must be paid to (i) running a close herd, (ii) the use of shared farm boundaries with double fenced, (iii) vaccinating susceptible livestock, (iv) using improved equipment and visitors' hygiene practices, (v) the maintenance of animal QoL to prevent disease transmission, (vi) do regular pest control, (vii) clean and disinfect vehicles after moving them, and (viii) minimize animal contact with neighbours, etc. These parameters offer a window of opportunity to use IoTT. As a data-oriented technique, a proper codifying process of existing and new parameters will be a valuable asset to use IoTT.

As the world recovers from the devastating damages caused by the COVID-19 pandemic, the place of data sharing capability on a wide scale to alert about new virus(s) of pandemic proportion is further justified [119]. For example, data sharing capability is key to using important biosecurity procedures such as the next-generation sequencing technique to alert stakeholders of the possible outbreak of novel zoonotic viruses before it is even isolated [120]. The role of IoTT in this will be to facilitate the collection of data and make them available for new procedures to report pandemics before it even occurs. Newer variables and metadata that are now known from the COVID-19 pandemic can be relied on to achieve effective surveillance through monitoring and control of zoonoses. The confluence of AI-ML and IoT will assist IoTT in its undertaking to monitor farm management practices to reduce risks from zoonoses and give more accurate zoonoses detection for predictive purpose. This implies that a key responsibility of IoT as a CPS and in synergy with ML to drive S\&DI is the provision of the technicality to automate LsM labor-intensive activities.

\subsection{Livestock Welfare and Product Quality Control}

There are IoTTs that exist in the literature, which are indicative of the potentials of IoT to manage the welfare of livestock and their product quality. The IoTTs were successfully tried to track animal activities using drones and classification techniques [121], monitor animal behaviour at an individualized level with automated analysis to decode animal welfare [31], and use an infrared thermal imaging technique to examine and detect stress and edema in animals [122], etc. This prospective justifies the possibility of adopting IoTTs to support LsM on a robust proportion. What IoTT requires is a codifiable template to leverage to make its impacts in disruptive proportion. This exists. For example, Figure 7 shows the standardized conceptual attributes, among others that are available to start with [123]. These standards have formed the basis to assess animal welfare and ascertain 
their level of wellbeing. Recent reports on animal welfare have highlighted these same attributes as how the quality of animal products are perceived [124]. This implies that there is a connection between animal welfare and the quality they imply as a product on the shelves in the meat market. This perception has also been linked to the poor quality of animal feeds and over-use of antibiotics. The job of IoTT is to leverage the operationalized form of these attributes to collect historical data from which livestock vitality is understood. Trends can also be identified and learned from these data to improve cattle welfare, their QoL, and by extension, product quality.

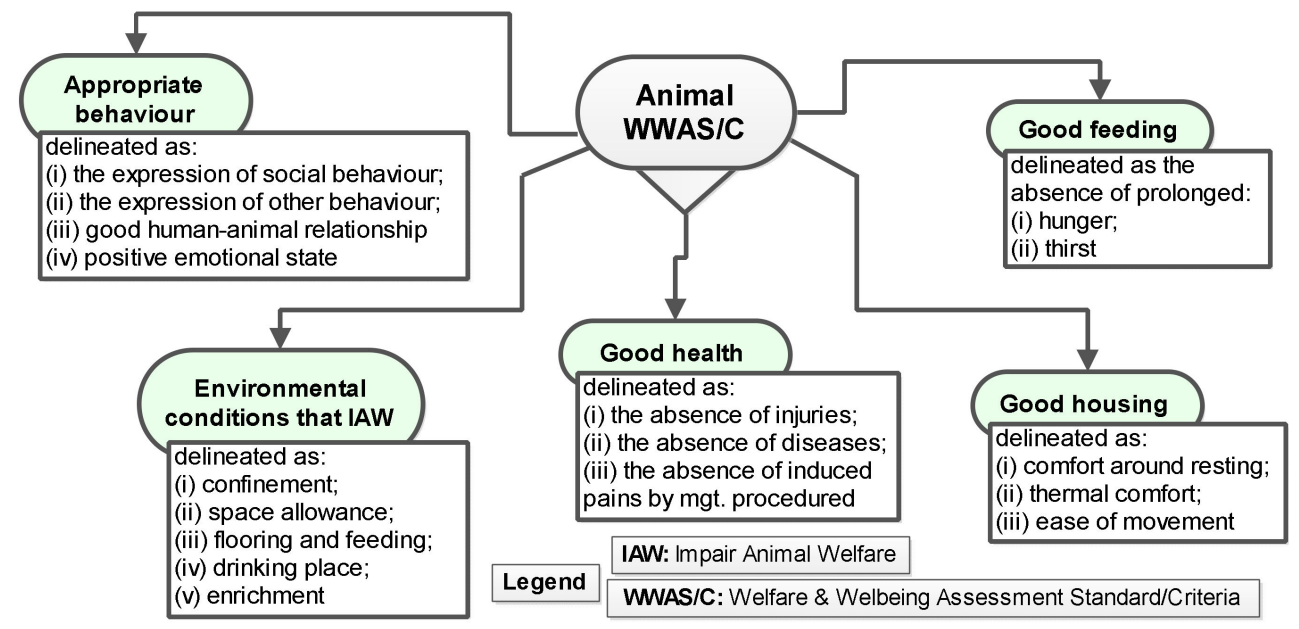

Figure 7. Standard principles for assessing animal welfare.

\subsection{Animal Recognition through Identification}

Livestock identification (Lst-Id) is an old tradition. It has been practiced for decades to manage livestock. It was initially meant to indicate animal ownership. However, as time progresses, it has been used to combat disease outbreaks [125]. Lst-Id is about keeping accurate livestock records that should start from when an animal joins the farm or from birth. Adequate knowledge about the health status of animals can be significantly relied on to influence animal's productivity. It can also help with a thorough elicitation of epidemiological information to the advantage of impacting animal welfare. Having unique animal identifiers have been found to support traceability. Identification plays an integral role in traceability. It provides the opportunity to trace individual animal species or breed for different reasons within the food supply chain.

Starting from the point of selling an animal or even at the point of its consumption back to when the animal's breeding happened even through to when it was slaughtered, an animal and its product can be traced. The true health status of an animal, which is tantamount to its quality at the time of consumption, can be unravelled. Traces of information (ToI) is what is required. The concept of digital tracing that allows the use of tracing points (i.e., ToI) makes tracing through sensors possible. The use of DNA has been the most useful procedure for this purpose. The vista traceability offers will take a new dimension that will open up substantial opportunities with the advent of IoTT. For example, IoT in confluence with the blockchain technology will influence traceability simultaneously with security and authenticity. These features offer limitless possibilities to monitor details concerning time, and the origin and quality of products. Traceability is linked with profit maximization and guarantees the safety and quality of products due to transparency. IoT will take this dimension to a new level by offering sensing data and technologies with connectivity options in LsM [40,126].

It is important to keep records of specific livestock species. The concept of identification will make sure this happens with the aid of IoT. This will support the easy remembrance of the species to re-order due to their potential value regarding meat quality and milk yield. Techniques in terms of algorithms, processing speed, and storage capacity 
are not hindrances nor lacking to achieve record keep for sundry purposes. IoTT as retrofits and in the LsM future means the possibility of using the potential of the big data and blockchain conception for data analytics and to incorporate transparency as part of modern farm management practices. IoTT is already positioned to make this happen, considering their existing potential to retrofit devices such as ear clippers, notches, taggers, barcode, QR code, facial recognition, micro-chipping, radio frequency identification (RFID), ear collar and tags with electronic capacity, and biometrics (e.g., muzzle prints, iris patterns, and retinal vascular devices), etc., with IoT sensors $[127,128]$. Some of these methods allow the permanent identification of animals on individual basis, unlike others that do so on temporal basis. RFID has fallen prey to reverse engineering. This notwithstanding the role of IoT and its complimentary technique such as the blockchain will make it difficult to compromise them when using in LsM [129]. Animal biometrics [128] and facial recognition [6] are both gaining attention as electronic means of animal identification due to their usage advantage. They suffice as simplified means of recording and retrieving flock information. Embracing this with IoT and its role will provide the platform to fast-track data-driven and inferential decision making anywhere and anytime regarding animal recognition through identification [130].

The use of animal yield potential and health status (i.e., QoL) as means of individual animal recognition is still new in the literature. Basically, this can be attributed to the traditional method that is unreliable and costly. The introduction of IoT in this aspect of LsM would make it possible to use the sensing potentials of IoTT. Compared to the traditional approach, IoT will make it possible to use Internet-based connectivity with appropriate sensing technologies. So, computing using IoT sensor technology will incorporate the use of non-manual methods to easily monitor the association of breeds, their health concerns, milk, and meat yield, etc. [131]. In [131], a deep-learning biometric-based approach was introduced to uniquely identify individual animals based on their yield potentials. In a related study, [132] showed the possibility of individual animal recognition, while in [6], this possibility incorporated remote sensing and monitoring technique to ascertain animal health and welfare status. This extent of success was before the advent of IoTT. The role of IoT will be the incorporation of the wherewithal to contextually recognize individual animals by means of identification, which was not considered in $[6,131,132]$. IoT will play this role, since it is customized to contextually recognize things through sensing. This implies that existing thermometers, microphones, and cameras can be retrofitted with sensors to remotely sense and monitor as well as recognize animals on an individual or group basis using their unique features such as body heat, motion, sound, and image $[6,128,131]$.

\section{IoT: Opportunities for Livestock Management}

In the management of livestock, the responsibility of IoT can be aggregated in its role to apply remotely controlled operations with advanced and enabled sensors and actuators for management advantages. In the IoT model of protocol stack data logging, transport, management, processing and reporting and security and privacy functionalities are enabled to work with sensors hardware for positioning, touch, proximity, vision, etc. This potential is what IoT will employ to play its management role that will make it possible to initiate precise intervention even purposefully in the context of sensitive circumstances in the management of livestock. The context sensitive nature of livestock management makes it imperative to seek the robust digital solution IoT offers. As the world enters the cusp of another ICT revolution, the use of IoT for LsM would offer lots of opportunities going by the role it will play. These opportunities can be understood through the lens of the drivers of the IoT revolution. For brevity due to space and time, two of the main drivers are considered, since they are the core technologies to drive the realization of the full potentials of IoT even in the livestock industry. 


\subsection{Cloud Computing and the 5G Nexus}

In the IoT context, devices will need to operate communally. Therefore, innovative applications that support such operation are needed [133]. The cloud platform is a driver/enabling technology that allows computing as a service over the Internet. This is one innovation that IoT will leverage in LsM. Existing LsM uses known capabilities as against the unlimited self-capabilities that IoT deploys that will yet get better with more data. This implies that as the volume of data grows this self-capability will also grow in the same proportion. The cloud will play a major role in the use of IoT unlimited selfcapability for LsM. Like other domains, LsM require services from cross-cutting domain to create innovative solutions. Cloud computing provides the essentials that IoT will need to coordinate the extremely heterogeneous resource capabilities that the modern LsM practice needs. This the traditional system of computation cannot provide. For example, the computing and storage services that befit the type of computational opportunities IoT will open up in LsM cannot be provided by the traditional computing and processing potentials. Additionally, as the livestock industry embraces the use of IoT paradigm, it will be inevitable to adopt the computing-infrastructure-as-a-service model. Farmers, especially in low income regions, would not need to own any infrastructure to use computing and storage services. In so far as the ability to connect to the cloud and pay a token exists, this service(s) will serve livestock farmers well. Based on cloud technology, the platform-asa-service, infrastructure-as-a-service, and software-as-a-service models would be easily available to livestock farmers.

Cloud computing will be driven by three enabling technologies: the Cloudlets, Fog computing, and mobile-edge computing (MEC) [134]. These technologies drive the service orientation of the cloud through the specific functions they perform. In the context of the management of livestock, the function of the Cloudlet would be to grant livestock farmers access to the cloud to use the computing-infrastructure-as-a-service model. Aside from this access opportunity, the usual end-to-end latency in cloud access would be managed by Cloudlet by reducing any inactivity. This is because high latency during cloud access could frustrate livestock farmers' experience during access to a cloud service. After access is granted, it is the responsibility of MEC to ensure that the computing power in the cloud is sustained without interruption due to lack of energy. While MEC takes up this responsibility, the function of Fog computing will be to ensure that the paradigm of distributed computing is operational and available for use by farmers. Fog computing will also promote the use of near-user edge devices' function to improve computation. This resource will afford farmers the open opportunity to share the many distributed routine tasks of monitoring with sensors on a regular basis even at odd times from remote location. As is, latency, the near-user edge device services by MEC (see Figure 8), the need for low energy computation, etc., that the existing 4G LTE network offers still need improvement. This is where the 5G (i.e., 5th Generation network) nexus connection comes in to provide the pragmatic way to connect in reality to the foregoing functionalities to deliver high-quality services.

A further look at MEC is justified by the computational need of the smart method of livestock management. For example, as depicted in Figure 8, data from mobile devices and other sundry devices are moved to MEC, where the computational tasks that is needed on the data are carried out. In case MEC encounters some unsolvable computational demand, the cloud will take over since it has superior (and more) computational resources. Basically, MEC's function is to ensure that the cloud's central computational function is present at the network edge, which is closer to users. As a consequence, operators fancy MEC and, thus, make it operate near the users to provide storage and computing services for them. A typical MEC architecture is presented in Figure 8 as a contribution in this paper. However, since detailed research into MEC is out of the scope of this review, readers are encouraged to consult related materials such as $[28,134,135]$ and many others for more information. With MEC, end-user clients are able to perform a massive amount of computation and storage in real time instead of doing so in the data centers that reside in the cloud. MEC support 
for latency, the direct access to real-time network information, and high bandwidth are the key advantages it seeks to contribute to the conception of managing livestock smartly. MEC's closeness to subscribers than the cloud through application program interfaces, its virtualised nature, and ability to locally manage its resources aggregately make it important to operators and user applications alike. All these potentials make it plausible justifiable to leverage it for the smart management of livestock.

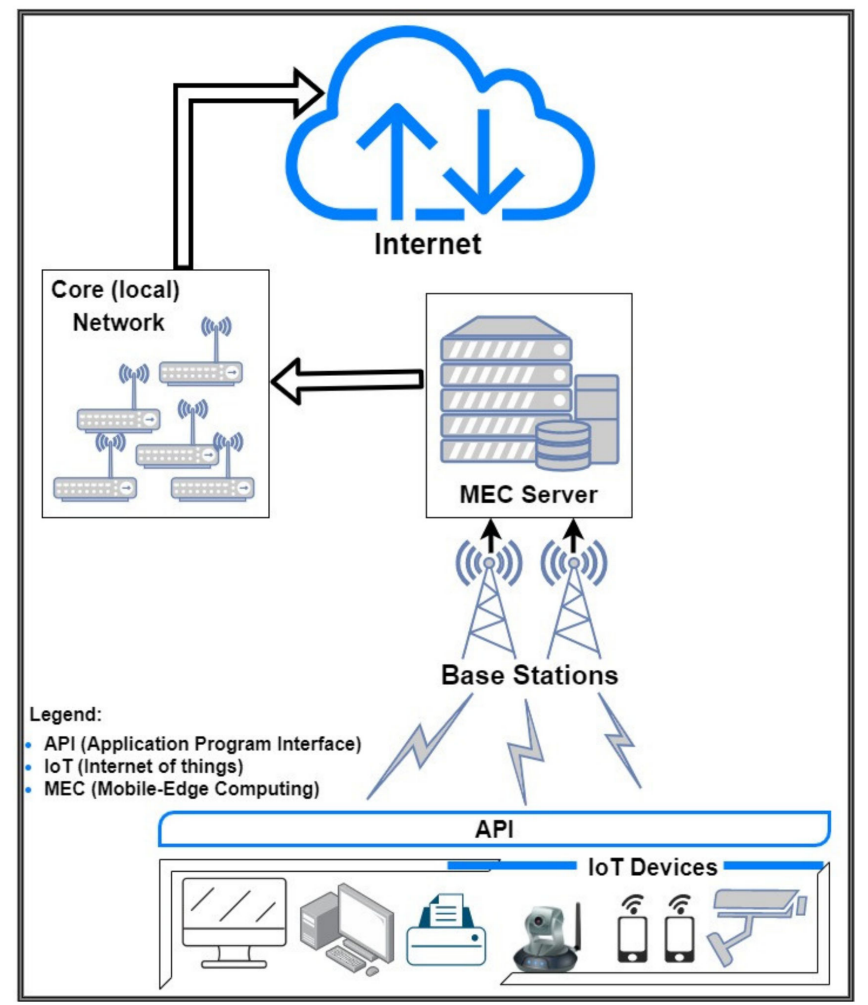

Figure 8. A typical mobile-edge computing (MEC) architecture.

The 5G network seeks to offer the nexus to provide additional strength for cloud computing by offering high spectral efficiency, which the current $4 \mathrm{G}$ network [28] is unable to provide. However, until $5 \mathrm{G}$ is officially revealed, $4 \mathrm{G}$ still remains the network to deliver the infrastructure for IoT to be driven by cloud computing to perform its role. Currently, the $4 \mathrm{G}$ network continues to struggle to satisfy the requirements of being a scalable network with ubiquitous communication coverage. As an infrastructure, its pricing and billing capabilities as well as the provision of backhaul connectivity and potential flexibility to manage IoT devices' performance with guaranteed security and privacy capabilities are still lagging behind. There is also the challenge of not being able to seamlessly offer network densification. With the 5G network, these challenges will be a thing of the past as a better generation of network. For IoTT to be deployed such that its potentials are fully utilized, the requirements highlighted earlier must be met, hence the 5G network is expected to fill this gap by supporting a super high network for superb communication [28]. The opportunities cloud computing and the 5G network offers would be extra-improved capacity to use computing resources. With this infrastructure in place, at a fee, owning computing infrastructure may not be necessary. LsM could come at the click of a button. Many of the connect-and-integrate-oriented livestock equipments after retrofitting with IoT-sensors will be connected easily to the cloud. Cloud-based IoT portal will also be available to provide a generic and specialized methodology to support sundry system integration to use the resources of the cloud to support the management of livestock [133]. 


\subsection{Disruptive Technologies}

Cloud computing and the 5G network are also part of the technologies with disruptive tendencies (DT). Their disruptive proportion is dependent on their capacity and based on their performance indicators that highlight their potential to defile known performance metrics (PM) of competing industries. Until the emergence of DTs, it was difficult to defile these PMs. However, though DTs do change the tone of the competitive edge of industries to newer levels of competition [136], for a technology to be innovatively disruptive, it must exhibit general purpose technological tendencies. This is the capability to provide alternative and sustained value. General purpose technological tendencies are pervasive and have broad application spectrum with the potential for continued technical advancements. Support for innovation is their hallmark with the strong tendency to handle repetitive tasks. They do this with better precision than humans. As "innovation complementarities", they open up many vistas for IoT to achieve its full potentials. Since the livestock industry is a niche industry, like others, the industry could rely on the disruptive potentials of DTs to its competitive advantage [137,138].

Arguably, big data, blockchain technology, and AI are other DTs that will compliment IoT to offer limitless opportunities in the management of livestock. Some of the opportunities expressed earlier were consequent upon the complimentary provisions of cloud computing, blockchain, and the 5G network to offer resources to support communal and high-power computing with security capabilities for IoT to leverage in LsM. Additionally, AI will contribute the techniques to drive analytics and inelastic computability as the brain of IoT. While IoT will be supported by big data to deliver the volume of data needed for sundry analytics, AI in synergy with big data will acquire the power in the data to unravel responses. These responses would offer the creativity and context to initiate smart and intelligent actions to manage livestock. Among the other role of the DTs in supporting IoT to play its managerial role is the clout the DTs will provide for IoT to be truly pervasive. This will provide a core nexus of influence for things to easily connect and promote the acceptable spread of IoTT applications to wide geometric proportion. In summary, the role of these DTs as general purpose technologies is that of "innovation complementarities". This will make them sufficiently resourceful to compliment IoTT. Additionally, in LsM, previously underutilized, untouched, and often ignored data will be exploited for decision making, since their potential power and use were poorly understood and the wherewithal to use them were unavailable until the unveiling of IoT [139].

\section{IoT's Adoption for Livestock Management}

This section presents a snippet of a future direction with highlights on the likely trends and future of LsM with IoT. IoT's managerial function is examined, therefore, vis-à-vis its key roles in LsM. Additionally presented is the likely impediments or barriers in IoT's adoption for LsM.

\subsection{The Future of Livestock Management with IoTT}

The future of IoT is already here. The success recorded with the use of IoT-based digital contact tracing capability during the current COVID-19 era to contain its spreads and also alert individuals of possible exposure highlighted two facts. Firstly, that it is now possible to use IoT-enabled devices to monitor infectious diseases. Secondly, that the technology to drive IoT for sundry management purposes in domains that are interested in the use of IoTT is here and advanced. For infectious disease detection through surveillance in various aspects, it is now possible to use IoT in confluence with tactile Internet and AI to propose novel solutions using new computational models that can drive the optimisation of productivity [139]. The future for IoTT in LsM is that that will require technologically advanced tools for communications, sensing, and computation. Of utmost significance is the possibility of exploring the fundamental resources IoT would offer to allow the design of IoT-based solutions in a real standardized way. Given the continuous advancements in the DTs presented earlier, IoTTs' automation would, therefore, be possible. 
Obviously, in the livestock industry, the foregoing should result in new ways of organizing, scheduling, and doing things, etc. The farmers of the IoT generation would need to adjust a lot of things. The entire livestock ecosystem and culture would be disrupted with the change that encourages all-around competitiveness. New maintenance strategies and skills will emerge to sustain different devices and pieces of equipment. These will lead to more challenges, some of which might be difficult to see now. Humans may even need to step aside from the scene if the right framework is not deployed to lay the groundwork for IoT's deployment in such a way that their relevance is still intact. All these present a whole new world of opportunities in which there will be new job roles where humans may even be confined to both moderators and overall controller, etc. Based on the findings in $[3-5,13,14,64]$, being proactive is important. This is because under the dispensation of using IoTT in LsM, the system in use will be autonomous, and actuators will be most efficiently instructed by sensors and optimised for sundry utilities and resource usage for better than how humans would do it.

Similarly, with IoTT, it will be possible to have smart milking stool, milk sieve/strainer, meat mincers, refrigerating system, feeders, waterers, chutes, calving pens, elastrator, and wagons, etc., that will interact with (e.g., talk to) farmers' smartphone and prompt them to initiate compensatory and/or spontaneous and follow up activities. This particular discernment will be enabled by concomitant data through devices that are IoT enabled. This is because these IoT empowered devices will be able to learn from observing-in this case-the behaviour of livestock and follow the preferences of stakeholders. These preferences will be checked against business insights. These insights will come from unlocked terabytes of analysed data from numerous touchpoints whenever connections are established. As [140] puts it, IoT-assisted devices would be able to demonstrate that they are engineered to understand and know a lot about different human behaviour might be scary. However, this is truly going to be the future, even for LsM. The opportunities that emerge as a result of data would be untold, and so long as there is connectivity, decision making will be efficiently improved, and market dynamics, end-user customer service experiences, and brand value addition, etc., will be huge.

\subsection{Key Roles of IoT in Livestock Management}

Based on the literature finding through a desk research following the practice in [141] by the review of deliverables from materials, projects, scientific conference, and journal paper publication, it was observed that it will be difficult to highlight the key role of IoT in one piece. This is due to its wide area of application. This also attest to IoT potentials based on available case studies of its capabilities [142]. So far, in this presentation, some of these roles have been presented. Nevertheless, a summary and recap of the roles are needed for quick reference by stakeholders. This inspires and supports the rationale to strive to overcome the impediments highlighted as a drawback to IoT adoption, particularly in the management of livestock. To put IoTT's key role in LsM in better perspective, its potential applications in related vertical industries are presented using the lens of IoT's features.

In some of the presentations already made in the literature, the role of IoT in specific contexts provides the lenses to highlight and understand the role. For example, [86] considered arable farming, and [142] highlighted theirs based on livestock and crop monitoring within the context of dairy farming using the perspective of intelligent edge-based IoT platform with the technology of blockchain. Basically in the LsM context IoT will play the key role of monitoring, forecasting, traceability, documentation, controlling, and the optimisation of logistics and navigation maneuvers, etc. An important aspect of the IoT key role is the platform it provides to implement multiple applications in the management of livestock. As found in [86], IoT's role can be best interpreted from three IoT concerns, i.e., the use of gateway in the network layer, sensing in the device layer, data processing and computing, and the use of GUI in the application layer. This can be extended to the processing and service management layer, thus countenancing the current IoT five layers. It is in the processing layer, which is separated from the network layer, that events are 
handled. To provide the means to act upon these events data, efficient database transactions are needed. In the service management layer, businesses such as the management of the whole IoT system are managed to include IoT applications and users' privacy (where the blockchain comes in, in recent formation), etc.

Drawing from the review of the literature, forecasting will engage different data sources by means of analytic methods to make concrete prediction of events. This is an important role that IoT will play along with monitoring where timely sensing of parameters of very diverse proclivities will be made. This role is particularly important, since it is the initial entry point for many other applications. These roles highlight the need for documentation to take care of the data sample that is needed for forecasting, monitoring, control, etc., for later use. In LsM, this will be important for management purposes, where traceability of products or produce is a regular occurrence. As for control, the role of active monitoring and the use of data to automatically control actuators after activating them are going to be daunting, whereas LsM will require the use of devices and sundry machines. Therefore, to use them in an organized way to improve the success of production, a different option of connectivity is required. This implies the activation of sundry operational processes (OP). This requires logistics and optimisation analytics, which IoT will provide to guide the OP to augment the daily routine of scheduling (for instance) in LsM. In Table 8, a succinct summary of the role of IoT in livestock management is presented.

Table 8. Additional IoT's role in livestock management (a summarized version of [37,71,142-147]).

\begin{tabular}{|c|c|}
\hline Role & Area of Application \\
\hline Monitoring & $\begin{array}{l}\text { Identifies animal location and movement. Monitors animal behaviour, } \\
\text { walking time, grazing time, resting time, and water or / and feed } \\
\text { consumption time, milk conductivity, animals' health by means of } \\
\text { monitoring animal temperature. Monitors animal activities such as physical } \\
\text { gesture recognition, heat stress, nutrition can also be monitored by } \\
\text { automatic observation. This also goes for rumination, heart rate, breath, and } \\
\text { in the detection and prevention of plagues, and in the reduction in the effect } \\
\text { of greenhouse gas. }\end{array}$ \\
\hline Controlling & $\begin{array}{l}\text { Takes control, responsibility, and account for vital nutrients in animal feeds. } \\
\text { It also facilitates automation, use in temperature control, etc. }\end{array}$ \\
\hline Forecasting & $\begin{array}{l}\text { Predicts the likelihood of future trends. Through prescriptive analytics how } \\
\text { future events are responded to base on data analysis are covered. }\end{array}$ \\
\hline Traceability/Tracking & $\begin{array}{l}\text { In supply and value chain. Animals' locations can be tracked and } \\
\text { monitored by means of activities to save thefts and wild attack. }\end{array}$ \\
\hline Documentation & $\begin{array}{l}\text { Plays the vital role of a store house to enable the processing of huge volume } \\
\text { of data. This has become possible, since IoT is correlated with turning out } \\
\text { data. It synergizes with cloud computing and big data resources to this. }\end{array}$ \\
\hline LO\&NM & $\begin{array}{l}\text { IoT is applied to achieve self-driving tractors and GPS field mapping, and } \\
\text { their sensors for unmanned ariel vehicles. }\end{array}$ \\
\hline Management & $\begin{array}{l}\text { Helps in making smarter decisions concerning animal health, weather, and } \\
\text { the production of quality product using data. Ensures the optimum usage of } \\
\text { farm materials, e.g., drugs, feed, and grain. It also aids their planning and } \\
\text { administration. Provides and manages scheduling of resources that are } \\
\text { restricted to enhance productivity, livestock conditions, farming principles, } \\
\text { and quality dairy products. }\end{array}$ \\
\hline Innovative initiator & $\begin{array}{l}\text { Provides platform for innovative and creative way to solve both in-field and } \\
\text { out-field problems. }\end{array}$ \\
\hline $\begin{array}{l}\text { Technical } \\
\text { responsibility }\end{array}$ & $\begin{array}{l}\text { Generates big data for edge computing. Ensures ingestion of data from } \\
\text { dissimilar heterogeneous sources. Allows interaction between sensors and } \\
\text { blockchain to support traceability. Provides cross-platform for devices to be } \\
\text { smarter and intelligent. Its gateway makes it possible for end-devices to } \\
\text { connect and allow stream data to the cloud for analysis from which issues } \\
\text { identified. Settle interoperability concerns among multiple operators. }\end{array}$ \\
\hline
\end{tabular}




\subsection{Barriers to IoT Adoption in Livestock Management}

As optimistic as some of the key roles of IoTTs are, which were explicated regarding the management of livestock (see Table 6), the challenge of data and device heterogeneity $(\mathrm{D} \& \mathrm{DH})$ still remains a huge impediment to tackle in the future. $\mathrm{D} \& \mathrm{DH}$ is the result of the high amount of variable processes and diverse scenarios that will require large heterogeneous IoT functionalities to operate. This challenge hampers the uptake of IoT at a large scale, of which the adoption scenario will also be the same in livestock farming. Recent research has shown that the challenge will be sorted out going by the existence of advanced technologies. In [148], this was shown by the architectural approach that was proposed to align system architectures with enabling reuse to model IoT-based systems, among others. Some of the other barriers to the adoption of IoTT in LsM as identified from the literature include, but are not limited to, the following. (1) How to coordinate and manage network complexity. This will result from the number of wired and wireless network connections vis-à-vis communicators and receivers that will be required per time? This means livestock farmer(s) will need to train, retrain, and/or hire experts to manage this challenge. Research efforts will also be needed to help document best practices to learn from and solve this problem. (2) The management of power amidst energy requirements. Though significant efforts have been made to tackle this challenge, more efforts are still needed to strike a balance between energy consumption, communication coverage, and data rate [2]. The target for balance should be the non-stop use of IoT devices when efficient sources of energy provision are achieved for sundry usages. (3) This third challenge is about how to leverage and integrate the advantages of the $5 \mathrm{G}$ network within the context of LsM. This is because of what the $5 \mathrm{G}$ network needs to functional properly. Like IoT, the 5G network operates with enabling technologies such as the massive multiple-input multiple-output technology, C-RAN, HetNets, mmWaves, and non-orthogonality. Their sole responsibility is to strengthen existing wireless sensor network infrastructure, though architectures and frameworks are needed to successfully adopt the 5G network enabling technologies.

The need to (i) understand the characteristics of distinct IoT traffic and (ii) maintain their complexities, which requires concerted research effort, are part of these challenges. The other challenges include the management of the overheads from time-critical IoT devices and delay intolerant services. This affects latency issues due to the operational diversity from distinct IoT technical activities $[6,28]$. At the level of connectivity, there will be mobile instability. This requires technical expertise to manage. It is compulsory to attend to it, since the challenge definitely come as a result of the sundry connectivity options, which IoT offers, and the need to constantly overhaul IoT infrastructure that changes continuously. These impediments have been identified as capable of limiting the efficient use of computing and communication resources by IoT devices. These technical bottlenecks are also making it difficult to develop intelligent and adaptive solutions that will use ML technique in diverse domains including the livestock industry [149]. The cost of data communication and long data access, especially in rural or isolated environments, are additional limitations to IoT adoption in LsM. In Africa, this is a huge challenge because a lot of cattle grazing takes place in rural and isolated environments. The cost of hardware, the challenge of long-range communication, the need for resilient design against environmental hazards, etc., in low-income countries are other barriers to worry about because they remain a big deal in the adoption of IoTs for rural access $[43,86,149]$.

\section{Cost Implication of IoT's Use in Livestock Management}

The technology of IoT is data and function driven. Therefore, to understand IoT's functionalities, its key elements present an interesting lens to do so (see Figure 6). These functionalities are representative evidence of the role IoT will play in LsM. Nevertheless, this role(s) comes at some costs. This is because every technology function at some cost [150]. In the literature, the conception of "cost implication" highlights monetary concerns, as well as time and energy (skill set). However, as IoTT gains and assumes new functions, its monetary cost drops proportionally, thus following the pattern identified by Moore's 
techno-economic model (MTeM) [151]. However, what is considered as cost implication in this article is the cost at which IoTT is deployed and used, since the monetary aspect is ultimately negligible going by MTeM. Therefore, the cost at which IoTT is deployed and used is tantamount to X-raying the contribution of the livestock farmer who is at the centre of ensuring that IoT use lives up to its functional expectation and promise. Hence, "cost implication" is herein referred to as the "cost at which IoT is to be used in LsM" (C-IoT-L). From this perspective, C-IoT-L is, thus, presented as the cost at which IoTT is coordinated and its services maintained in LsM by livestock farmers. This the authors also conceptualises as the time and energy (skill set) deployed by a farmer to ensure the sustainability of IoT.

IoT's six elements that are representatives of its capabilities [57] present a first step to also consider C-IoT-L. However, despite available knowledge about these features (i.e., elements), it is only the feature of "identification" that has been appraised regarding C-IoT-L, hence the need for this appraisal. However, the existing appraisal presents a template of some sort to consider C-IoT-L, since unarguably, the six features of IoT show the aggregate of technicalities that will be needed in a farmer to interact with different IoTs. This cost by implication and in the context of consideration is at the level of the farmers hard earned experiences over time. This type of cost is often unquantifiable and operationally going by the provisions in the literature; the dynamics of estimating it is tricky. It is also because of the continuous changing nature of IoT infrastructure that is derived from a changing implementation framework. This has an implication too in that until a particular IoT solution is working, estimating its C-IoT-L in the context of this paper vis-à-vis the contribution of farmers may not be feasible. Hence, the focus is on a much broader aspect of qualitatively appraising C-IoT-L. This affects the societal and behavioural adjustments and the innate sense of farmers that mature over time into an experience-based skill set (EbSS). This is leveraged to show the C-IoT-L through the lens of time and energy (skill set) that farmers put into LsM.

IoT is a digital task and burden reliever. It plays this role by accepting the responsibility of knowing things by sensing for instance what mix of feed and combination of drug are to be administered as its contribution to animal welfare. With this responsibility comes the issue of animal care culture. This culture in the conventional livestock farming practice allows animals to be cared for on a one-to-one basis. In this one-to-one-care culture, there is the characteristic bonding that fosters relationships between human and animals. This care culture would definitely be at risk as IoT takes over tasks that encourage bonding. This will come at the cost of redefining the whole conception of "care concerns for animal wellbeing". There is evidence that shows that levels of human bias exist in the culture of animal care. Yes, the use of technology may reduce this bias, since they are able to work uncompromisingly regarding empathy, sympathy, and compassion unlike humans' who are subject to mood swings [85]. However, the tradeoff of non-human involvement is at a huge cost, which is the eroding the bonding between human and animals.

Another aspect to look regarding C-IoT-L is in respect of the digital platform IoT presents by which it will play the role of offering standardized systems of animal welfarism to uphold and sustain high levels of concern (i.e., respect) for animals at the same level $[85,123,152]$. This task is highly repetitive and utilitarian, but the involvement of data will make it easy for IoT to carry out. Nevertheless, though this approach to animal welfarism upholds the "aspect of respect" for several animals at the same level, it is actually at the cost of the absence of the "one-to-one-care culture" and its characteristic bonding. This cost is unquantifiable too. While the tasks of automating the systems of animal welfarism may be quantifiable from the perspective of the cost of buying hardware and software to support the automation, the effort of the farmer in ensuring the system works is an additional cost to consider. Notwithstanding, the IoT must handle and sustain the "respect" aspect of animal care culture as much as data can afford; the exact cost of doing so is still at the expense of someone. 
Furthermore, the perspective of having IoTT devices operate in the farm presents yet another dimension to consider the C-IoT-L. During the discharging of the observatory and sensing role of IoT in the farm, feedbacks would be inevitable and prompt actions will be demanded. These feedbacks may be sent as an alert for farmers' immediate response. Definitely, actuators will not initiate all of the demanded actions or responses. Some very crucial ones would require the attention of farmers' who would deploy their innate skills to perform such actions. To rely on these skills implies they exist and are innately nurtured over time through cognitive learning and knowledge acquisition to play their role satisfactorily. Experiences of these nature and other similar ones are nuanced and highly subjective. They are also unquantifiable because they are innate senses that rely on judgements and accumulated knowledge, information, etc. These are EbSS, and as such, they offer deductive, inductive, and at the same time, inferential resources to make decisions when applied. These skill sets should be made to compliment whatever IoT delivers. This must be deliberately considered and recognized and given a place when formulating the implementation framework that will drive the continuously changing IoT infrastructure.

To discount the EbSS of livestock farmers due to the role IoT seeks to play in livestock management will come at a cost that may be detrimental to the whole concept of PLF. For instance, the EbSS of farmers will be relied on to identify and understand the problems thrown up by data sensors at different times in the livestock farm. The EbSS will also be relied on to create new ideas to analyse, synthesise, and make sense of IoT data and do follow up predictions using unbiased data validation generated from the learning curve of experience [6]. Farmers will be armed with this experience to live up to the requirements to adopt the right decision options that IoTT will throw up. These complementary roles are critical and must not be discountenanced, since they are from soft technical skills, as documented in the literature [153]. Based on the literature, IoTTs as service-oriented technologies will exhibit a profitable degree of technological advancements. However, it is noteworthy to emphasize that the onus will still be on humans to harness the intelligent solutions, which IoT will provide in LsM. This human intervention will come at an unquantifiable cost too. Its overall value addition may be estimatable, perhaps cognizant of the technologically innovative and creative role of IoTT vis-à-vis farmers' role in LsM. Some parameters may suffice for this estimation: the change in the management dynamics of what obtains in the conventional sense, the demand for what IoTT has to offer, and the potential to add value by employees' EbSS in the livestock industry. These suggestively may be good enough parameters for scientific investigation to ascertain what offers the most cost implication for development.

\section{Conclusions}

In this paper, a PRISMA-based systematic review of IoTT's use in LsM has been presented with LsM highlighted as a methodical process with strict regimentations. This provided the rationale to consider a highly logical and at the same time methodical technology such as IoT for LsM. IoT as highlighted so far will play a key management role in LsM. This will happen by deploying its primary feature of identification by sensing and observation, etc., through monitoring and control to manage the strict regimentations in LsM. The health and wellbeing of livestock have been highlighted as important factors in determining the quality of animals as products in the market. In some ways, the determination of animal wellbeing and health is still heavily dependent on human discipline, which is fraught with errors from misrepresentation and misinterpretations due to fatigue. However, the authors argued that the role of IoT in LsM for the same purpose is important in that its potentials will orchestrate profitable change. In this paper, the authors also justified their motivation that the burgeoning population of the world cannot survive on the traditional method of LsM practice by encouraging a rethink of the traditional process in favour of the use of IoT. This also raises the question of whether the livestock industry is ready to work in synergy with other proponents of IoT to surmount the barriers highlighted 
as impediments to IoT adoption in LsM. The cost implication of using IoT was discussed while highlighting with caution the need to uphold the place of farmers EbSS in the use of IoT in LsM.

Aside from the barriers inhibiting the adoption of IoT in LsM, there are non-adoption challenges. These challenges include greenhouse gas emission with livestock being a major contributor, the continuously dwindling land resource due to deforestation, and desertification for which sustainability has become a crucial issue to meet the upward demand for animal food in the world. There have also been biosecurity and zoonotic outcries that have been reinforced by the current need to forestall future pandemic such as the current COVID-19. These challenges and others make the LsM of the 21st century a very challenging endeavour. To live up to this challenge, this article attempts to draw stakeholders' attention to the use of IoT based on the role it can play to surmount the challenges. That IoT is correlated with data and also synergizes with DTs such as cloud computing, big data, and AI, etc., makes it imperative to try IoTT in LsM. The business insights it will unravel to innovatively create solution for both in-field and out-field problems is a testament to this.

Three factors make IoT uniquely fit to provide the wherewithal to take on the challenges confronting the endeavour of LsM in the 21st century. These factors are: IoT's retrofitting capability, its data-savvy nature, and ability to work in synergy with DTs (e.g., cloud computing, blockchain technology, AI, etc.). Technology often thrives innovatively when data are available. Big data, data mining, and other data-savvy endeavours confirm this. One additional unique role IoTT will play in LsM is the provision of limitless Internet connectivity options to harness the DTs to use data and, thus, support the delivery of sustained value. To this end, the rationale for IoT architecture, ecosystems, and other IoT concepts and enabling technology with cost implication among others were presented to help understand the management role of IoT in LsM and the nature of solution to expect, etc. Stakeholders within the livestock industry must show action-oriented interest in the use of IoT techniques for disruptive innovation to be realised in the livestock industry. This interest, the authors believe, can drive the optimism that is required to surmount the challenges highlighted as barriers to IoT's adoption in LsM.

As future work, a gap analysis is intended to investigate a range of relevant initiatives that includes the adoption of industry 4.0 technologies for extensive livestock farming to find if sustainability can be ensured through big-data-driven systems using IoT sensing networks, DL, and other ML-assisted techniques. Contributions would also be made in respect of the provision of a reference framework that adds additional functionalities to actualise CPS for extensive livestock farming going by the consistency observed in the literature $[92,99]$ regarding the use of digitalization to enforce sustainable productivity.

Funding: This research was funded by TETFund grant number 2012/2013 (Merged) TETFund ASTD.

Institutional Review Board Statement: Not applicable.

Informed Consent Statement: Not applicable.

Data Availability Statement: Data sharing is not applicable to this article.

Acknowledgments: This research work was supported by TETFund-under the supervision of Obafemi Awolowo University, Ile-Ife, Nigeria—and the University of the West of England, Bristol United Kingdom.

Conflicts of Interest: The authors declare no conflict of interest.

\section{References}

1. Guillen, J.; Natale, F.; Carvalho, N.; Casey, J.; Hofherr, J.; Druon, J.-N.; Fiore, G.; Gibin, M.; Zanzi, A.; Martinsohn, J.T. Global seafood consumption footprint. Ambio 2019, 48, 111-122. [CrossRef]

2. Navarro, E.; Costa, N.; Pereira, A. A systematic review of IoT solutions for smart farming. Sensors 2020, 20, 4231. [CrossRef]

3. Jamali, M.A.J.; Bahrami, B.; Heidari, A.; Allahverdizadeh, P.; Norouzi, F. Some Cases of Smart use of the IoT. In Towards the IoT; Springer: Cham, Switzerland, 2020; pp. 85-129. [CrossRef]

4. Pokorni, S. Reliability and Availability of the IoT. Mil. Tech. Cour. 2019, 67, 588-600. 
5. Casadei, R.; Fortino, G.; Pianini, D.; Russo, W.; Savaglio, C.; Viroli, M. Modelling and simulation of opportunistic IoT services with aggregate computing. Future Gener. Comput. Syst. 2019, 91, 252-262. [CrossRef]

6. Benjamin, M.; Yik, S. Precision Livestock Farming in Swine Welfare: A Review for Swine Practitioners. Animals 2019, 9, 133. [CrossRef]

7. Banhazi, T.M.; Lehr, H.; Black, J.L.; Crabtree, H.; Schofield, P.; Tscharke, M.; Berckmans, D. Precision livestock farming: An international review of scientific and commercial aspects. Int. J. Agric. Biol. Eng. 2012, 5, 1-9.

8. Berckmans, D. Precision livestock farming technologies for welfare management in intensive livestock systems. Rev. Sci. Tech. 2014, 33, 189-196. [CrossRef]

9. Nasirahmadi, A.; Edwards, S.A.; Matheson, S.M.; Sturm, B. Using automated image analysis in pig behavioural research: Assessment of the influence of enrichment substrate provision on lying behaviour. Appl. Anim. Behav. Sci. 2017, 196, 30-35. [CrossRef]

10. Lee, I. The IoT for Enterprises: An Ecosystem, Architecture, and IoT Service Business Model. Internet Things 2019, 7, 100078, 1-13. [CrossRef]

11. Internet of Things: Introduction to IoT Ecosystem-A Technical, Architectural, \& Solution Design Overview. Available online: https:/ / Medium.Com/Technology-Hits/Introduction-To-Iot-Ecosystem-25b359c8cf23 (accessed on 4 February 2021).

12. Enabling the IoT Ecosystem with Policy and Regulation. Available online: https://www.synergyconsulting.ae/insights/enablingiot-ecosystem-policy-regulation/ (accessed on 4 February 2021).

13. Ray, P.P. A survey on Internet of Things architectures. J. King Saud Univ.-Comput. Inf. Sci. 2018, 30, $291-319$.

14. Shi, X.; An, X.; Zhao, Q.; Liu, H.; Xia, L.; Sun, X.; Guo, Y. State-of-the-Art IoT in Protected Agri. Sensors 2019, 19, 1833. [CrossRef]

15. Eisler, M.C.; Lee, M.R.F.; Tarlton, J.F.; Martin, G.B.; Beddington, J.; Dungait, J.A.J.; Greathead, H.; Liu, J.; Mathew, S.; Miller, H.; et al. Agriculture: Steps to sustainable livestock. Nat. News. 2014, 507, 32-34. [CrossRef]

16. What Is Global Warming? Available online: https:/ / www.livescience.com/37003-global-warming.html (accessed on 22 July 2019 ).

17. Gebrekidan, T.; Guo, Y.; Bi, S.; Wang, J.; Zhang, C.; Wang, J.; Lyu, K. Effect of index-based livestock insurance on herd offtake: Evidence from the Borena zone of southern Ethiopia. Clim. Risk Manag. 2019, 3, 67-77. [CrossRef]

18. Hoy, M.B. The "IoTs": What it is and what it means for lib. Med. Ref. Serv. Quart. 2015, 34, 353-358. [CrossRef] [PubMed]

19. Ferreira, J.; David, J.M.N.; Braga, R.; Campos, F.; Stroele, V.; De Aguiar, L. Supporting the Collaborative Research through Semantic Data Integration. In Proceedings of the 2019 IEEE 23rd International Conference on Computer Supported Cooperative Work in Design (CSCWD), Porto, Portugal, 6-8 May 2019; IEEE: Piscataway, NJ, USA, 2019; pp. 319-324.

20. Bär, S.; Reinhold, O.; Alt, R. The Role of Cross-Domain Use Cases in IoT-A Case Analysis. In Proceedings of the Proceedings of the 52nd Hawaii International Conference on System Sciences, Grand Wailea, Maui, HI, USA, 8-11 January 2019; pp. 390-399. [CrossRef]

21. Asghari, P.; Rahmani, A.M.; Javadi, H.H.S. IoT applications: A systematic review. Comput. Netw. 2019, 148, 241-261. [CrossRef]

22. Zhang, X.M. Application of Internet of Things technology in agricultural production. In Proceedings of the International Symposium on Big Data and Artificial Intelligence, Hong Kong, China, 29-30 December 2018; ACM: New York, NY, USA; pp. 269-274.

23. Saeed, N.; Alouini, M.S.; Al-Naffouri, T.Y. Towards the Internet of X-things: New Possibilities for Underwater, Underground, and Outer Space Explo. arXiv 2019, arXiv:1903.11996.

24. Akyildiz, I.F.; Kak, A. The Internet of Space Things/CubeSats: A ubiquitous cyber-physical system for the connected world. Comput. Netw. 2019, 150, 134-149. [CrossRef]

25. Borgia, E. The Internet of Things vision: Key features, applications and open issues. Comput. Commun. 2014, 54, 1-31. [CrossRef]

26. Saheb, T.; Izadi, L. Paradigm of IoT big data analytics in the healthcare industry: A review of scientific literature and mapping of research trends. Telemat. Inform. 2019, 41, 70-85. [CrossRef]

27. Gil, D.; Johnsson, M.; Mora, H.; Szymański, J. Review of the complexity of managing big data of the Internet of Things. Complexity 2019. [CrossRef]

28. Agiwal, M.; Saxena, N.; Roy, A. Towards connected living: 5G enabled IoT. IETE Tech. Rev. 2019, 36, 190-202. [CrossRef]

29. Tabbah, R.; Maritz, A. Demystifying Disruptive Innovation Phenomenon: Economic and Societal Impacts. Rev. Cercet. Interv. Sociala 2019, 64, 9. [CrossRef]

30. Nord, J.H.; Koohang, A.; Paliszkiewicz, J. The IoT: Review and Theoretical Framework. Expert Syst. Appl. 2019, 133, 97-108. [CrossRef]

31. Rahman, A.F.A.; Daud, M.; Mohamad, M.Z. Securing sensor to cloud ecosystem using IoT security framework. In Proceedings of the International Conference on Internet of Things and Cloud Computing, Cambridge, UK, 22-23 March 2016; ACM: New York, NY, USA, 2016; pp. 79-84.

32. Data Acquisition Using Arrowhead Framework for Condition Based Maintenance of Industrial Equipment. Available online: https:/ / www.diva-portal.org/smash/get/diva2:1360493/FULLTEXT01.pdf (accessed on 5 February 2021).

33. Varga, P.; Blomstedt, F.; Ferreira, L.L.; Eliasson, J.; Johansson, M.; Delsing, J.; De Soria, I.M. Making system of systems interoperable-The core components of the arrowhead framework. J. Netw. Comput. Appl. 2017, 81, 85-95. [CrossRef]

34. Ammar, M.; Russello, G.; Crispo, B. Internet of Things: A survey on the security of IoT frameworks. J. Inf. Secur. Appl. 2018, 38, 8-27. [CrossRef] 
35. Marcu, I.; Suciu, G.; Bălăceanu, C.; Vulpe, A.; Drăgulinescu, A.-M. Arrowhead Technology for Digitalization and Automation Solution: Smart Cities and Smart Agriculture. Sensors 2020, 20, 1464. [CrossRef]

36. Kozma, D.; Varga, P.; Larrinaga, F. Data-driven Workflow Management by utilising BPMN and CPN in IIoT Systems with the Arrowhead Framework. In Proceedings of the 2019 24th IEEE International Conference on Emerging Technologies and Factory Automation, Zaragoza, Spain, 10-13 September 2019; IEEE: New York, NY, USA, 2019; pp. 385-392.

37. Alshohoumi, F.; Sarrab, M.; AlHamadani, A.; Al-Abri, D. Systematic review of existing IoT architectures security and privacy issues and concerns. Int. Jour. Adv. Comp. Sci. Appl. 2019, 10, 232-251. [CrossRef]

38. Santos, M.G.D.; Ameyed, D.; Petrillo, F.; Jaafar, F.; Cheriet, M. Internet of Things Architectures: A Comparative Study. arXiv 2020, arXiv:2004.12936.

39. Amin, F.; Ahmad, A.; Sang Choi, G. Towards trust and friendliness approaches in the social Internet of Things. Appl. Sci. 2019, 9, 166. [CrossRef]

40. Zhu, L.; Gai, K.; Li, M. Blockchain and IoT. In Blockchain Technology in IoT of Things; Springer: Cham, Switzerland, 2019; pp. 9-28.

41. Ma, Y.; Ping, K.; Wu, C.; Chen, L.; Shi, H.; Chong, D. Artificial Intelligence powered IoT and smart public service. Libr. Hi-Tech 2019. 0737-8831. [CrossRef]

42. Ngu, A.H.; Gutierrez, M.; Metsis, V.; Nepal, S.; Sheng, Q.Z. IoT middleware: A survey on issues and enabling technologies. IEEE Internet Things J. 2017, 4, 1-20. [CrossRef]

43. Akpakwu, G.A.; Silva, B.J.; Hancke, G.P.; Abu-Mahfouz, A.M. A survey on 5G networks for the IoT: Communication technologies and challenges. IEEE Acc. 2017, 6, 3619-3647. [CrossRef]

44. Shaikh, F.K.; Zeadally, S.; Exposito, E. Enabling technologies for green IoT. IEEE Syst. J. 2015, 11, 983-994. [CrossRef]

45. Manogaran, G.; Varatharajan, R.; Lopez, D.; Kumar, P.M.; Sundarasekar, R.; Thota, C. A new architecture of Internet of Things and big data ecosystem for secured smart healthcare monitoring and alerting system. Future Gen. Comput. Syst. 2018, 82, 375-387. [CrossRef]

46. Nikoui, T.S.; Rahmani, A.M.; Balador, A.; Javadi, H.S. Internet of Things architecture challenges: A systematic review. Int. J. Commun. Syst. 2020, e4678. [CrossRef]

47. Xu, H.; Yu, W.; Griffith, D.; Golmie, N. A survey on industrial Internet of Things: A cyber-physical systems perspective. IEEE Acc. 2018, 6, 78238-78259. [CrossRef]

48. Paniagua, C.; Delsing, J. Industrial Frameworks for Internet of Things: A Survey. IEEE Syst. J. 2020, 1-11. [CrossRef]

49. Hatcher, W.G.; Qian, C.; Gao, W.; Liang, F.; Hua, K.; Yu, W. Towards Efficient and Intelligent Internet of Things Search Engine. IEEE Acc. 2021, 9, 15778-15795. [CrossRef]

50. Gao, X.; Pishdad-Bozorgi, P.; Shelden, D.R.; Tang, S. Internet of Things Enabled Data Acquisition Framework for Smart Building Applications. J. Constr. Eng. Manag. 2021, 147, 04020169-1-04020169-15. [CrossRef]

51. Watanobe, Y.; Yaguchi, Y.; Miyaji, T.; Yamada, R.; Naruse, K. Data Acquisition Framework for Cloud Robotics. In Proceedings of the 2019 IEEE 10th International Conference on Awareness Science and Technology (iCAST), Morioka, Japan, 23-25 October 2019; IEEE: New York, NY, USA, 2019; pp. 1-7.

52. Kang, J.J.; Dibaei, M.; Luo, G.; Yang, W.; Haskell-Dowland, P.; Zheng, X. An Energy-Efficient and Secure Data Inference Framework for Internet of Health Things: A Pilot Study. Sensors 2021, 21, 312. [CrossRef]

53. Kaur, P.; Garg, R. A survey on key enabling technologies towards 5G. In IOP Conference Series: Materials Science and Engineering; IOP Publishing: Bristol, UK, 2021; Volume 1033, p. 012011. [CrossRef]

54. Varga, P.; Peto, J.; Franko, A.; Balla, D.; Haja, D.; Janky, F.; Soos, G.; Ficzere, D.; Maliosz, M.; Toka, L. 5G support for Industrial IoT Applications-Challenges, Solutions, and Research gaps. Sensors 2020, 20, 828. [CrossRef] [PubMed]

55. Popovski, P.; Trillingsgaard, K.F.; Simeone, O.; Durisi, G. 5G wireless network slicing for eMBB, URLLC, and mMTC: A communication-theoretic view. IEEE Acc. 2018, 6, 55765-55779. [CrossRef]

56. Li, S.; Da Xu, L.; Zhao, S. The IoT: A survey. Inf. Syst. Front. 2015, 17, 243-259. [CrossRef]

57. Al-Fuqaha, A.; Guizani, M.; Mohammadi, M.; Aledhari, M.; Ayyash, M. Internet of things: A survey on enabling technologies, protocols, and applications. IEEE Commun. Surv. Tutor. 2015, 17, 2347-2376. [CrossRef]

58. Shin, M.; Paik, W.; Kim, B.; Hwang, S. An IoT Platform with Monitoring Robot Applying CNN-Based Context-Aware Learning. Sensors 2019, 19, 2525. [CrossRef]

59. The Complete List of Wireless IoT Network Protocols. Available online: https://www.link-labs.com/blog/complete-list-iotnetwork-protocols (accessed on 26 July 2019).

60. Manufacturing of IoT Sensors Electronics \& Hardware. Available online: https://cdn.vibrantgujarat.com/website/ writereaddata/images/pdf/project-profiles/Internet-of-Things-Sensors.pdf (accessed on 1 August 2019).

61. Gajjar, M.J. Mobile Sensors and Context-Aware Computing; Morgan Kaufmann Publishers: Cambridge, MA, USA, 2017.

62. A Primer on the Technologies Building the IoT. Available online: https://www2.deloitte.com/content/dam/insights/us/ articles/iot-primer-iot-technologies-applications/DUP_1102_InsdeTheInternetOfThings.pdf (accessed on 2 July 2019).

63. DZONE: IoT Systems: Sensors and Actuators. Available online: https://dzone.com/articles/iot-systems-sensors-and-actuators (accessed on 1 October 2019).

64. Adams-Progar, A.; Fink, G.; Walker, E.; Llewellyn, D. Security and privacy issues in the Internet of cows. In Security and Privacy in Cyber-Physical Systems: Foundations, Principles, and Applications; Song, H., Fink, G.A., Jeschke, S., Eds.; John \& Wiley: Chichester, UK, 2018; pp. 375-398. 
65. Jayathilaka, W.A.D.M.; Qi, K.; Qin, Y.; Chinnappan, A.; Serrano-García, W.; Baskar, C.; Wang, H.; He, J.; Cui, S.; Thomas, S.W.; et al. Significance of nanomaterials in wearables: A review on wearable actuators and sensors. Adv. Mat. 2019, 31, 1805921. [CrossRef]

66. Bechar, A.; Vigneault, C. Agricultural robots for field operations: Concepts and components. Biosyst. Eng. 2016, 149, 94-111. [CrossRef]

67. Biggs, P.; Garrity, J.; LaSalle, C.; Polomska, A.; Pepper, P.; Husle, M.; Madigan, M.C.; Borno, R. Harnessing the IoT for Global Development. Available online: https://www.sbs.ox.ac.uk/cybersecurity-capacity/system/files/Harnessing-IoT-GlobalDevelopment.pdf (accessed on 2 July 2019).

68. Cirillo, F.; Wu, F.J.; Solmaz, G.; Kovacs, E. Embracing the Future Internet of Things. Sensors 2019, 19, 351. [CrossRef]

69. Loughlin, C. Sensors for Industrial Inspection; Springer Science \& Business Media: Berlin, Germany, 2012.

70. Thakkar, D.; Rajput, P.; Dubey, R.; Parekh, R. Design and Implementation of Autonomous UAV Tracking System Using GPS and GPRS. In Progress in Advanced Computing and Intelligent Engineering; Springer: Singapore, 2019; pp. $433-439$.

71. Kayacan, E.; Kayacan, E.; Ramon, H.; Saeys, W. Towards Agrobots: Identification of the yaw dynamics and trajectory tracking of an autonomous tractor. Comput. Electron. Agric. 2015, 115, 78-87. [CrossRef]

72. Hostiou, N.; Fagon, J.; Chauvat, S.; Turlot, A.; Kling-Eveillard, F.; Boivin, X.; Allain, C. Impact of precision livestock farming on work and human-animal interactions on dairy farms. A review. Biotechnol. Agron. Société Environ./Biotechnol. Agron. Soc. Environ. 2017, 21, 268-275.

73. Chadil, N.; Russameesawang, A.; Keeratiwintakorn, P. Real-time tracking management system using GPS, GPRS and Google earth. In Proceedings of the 5th International Conference on Electrical Engineering/Electronics, Computer, Telecommunications and Information Technology, Krabi, Thailand, 14-17 May 2008; IEEE: Piscataway, NY, USA, 2008; Volume 1, pp. $393-396$.

74. Mannini, A.; Sabatini, A.M. Machine learning methods for classifying human physical activity from on-body accelerometers. Sensors 2010, 10, 1154-1175. [CrossRef]

75. Shaeffer, D.K. MEMS-i sensors: A tutorial overview. IEEE Commun. Mag. 2013, 51, 100-109. [CrossRef]

76. Mandal, N.; Kumar, B.; Sarkar, R.; Bera, S.C. Design of a flow transmitter using an improved inductance bridge network and rotameter as sensor. IEEE Trans. Instrum. Meas. 2014, 63, 3-3136. [CrossRef]

77. Wang, X.; Zhao, X.; He, Y.; Wang, K. Cough sound analysis to assess air quality in commercial weaner barns. Comput. Electron. Agric. 2019, 160, 8-13. [CrossRef]

78. Park, J.H.; Kim, D.K.; Kim, H.J.; Song, S.J.; Cho, S.H. Development of EMA transducer for inspection of pipelines. J. Mech. Sci. Technol. 2017, 31, 5209-5218. [CrossRef]

79. Chai, H.; Phung, B.T.; Mitchell, S. Application of UHF Sensors in Power System Equipment for Partial Discharge Detection: A Review. Sensors 2019, 19, 1029. [CrossRef]

80. Jacobs, W.W.; Star, C. STAR calorimetry. J. Phys. Conf. Ser. 2009, 160, 012008. [CrossRef]

81. Babrauskas, V. The cone calorimeter. In SFPE Handbook of Fire Protection Engineering; Springer: New York, NY, USA, 2016; pp. 952-980.

82. Fang, X.; Bando, Y.; Liao, M.; Gautam, U.K.; Zhi, C.; Dierre, B.; Liu, B.; Zhai, T.; Sekiguchi, T.; Koide, Y.; et al. Single-Crystalline ZnS Nanobelts as Ultraviolet-Light Sensors. Adv. Mater. 2009, 21, 2034-2039. [CrossRef]

83. Sans, J.A.; Manjón, F.J.; Pereira, A.L.J.; Gómez-Tejedor, J.A.; Monsoriu, J.A. Oscillations studied with the smartphone ambient light sensor. Eur. J. Phys. 2013, 34, 1349. [CrossRef]

84. Del Villar, I.; Arregui, F.J.; Zamarreño, C.R.; Corres, J.M.; Bariain, C.; Goicoechea, J.; Elosua, C.; Hernaez, M.; Rivero, P.J.; Socorro, A.B. Optical sensors based on lossy-mode resonances. Sens. Actuators B Chem. 2017, 240, 174-185. [CrossRef]

85. Bos, J.M.; Bovenkerk, B.; Feindt, P.H.; van Dam, Y.K. The Quantified Animal: Precision Livestock Farming and the Ethical Implications of Objectification. Food Ethic 2018, 2, 77-92. [CrossRef]

86. Villa-Henriksen, A.; Edwards, G.T.; Pesonen, L.A.; Green, O.; Sørensen, C.A.G. IoT in arable farming: Implementation, applications, challenges and potential. Biosyst. Eng. 2020, 191, 60-84. [CrossRef]

87. Moher, D.; Liberati, A.; Tetzla, J.; Altman, D.G. Preferred Reporting Items for Systematic Reviews and Meta-Analyses: The PRISMA Statement. Clin. Epidemiol. 2009, 62, 1006-1012. [CrossRef] [PubMed]

88. Yao, X.; Zhou, J.; Lin, Y.; Li, Y.; Yu, H.; Liu, Y. Smart manufacturing based on cyber-physical systems and beyond. J. Intell. Manuf. 2019, 30, 2805-2817. [CrossRef]

89. Gupta, M.; Abdelsalam, M.; Khorsandroo, S.; Mittal, S. Security and privacy in smart farming: Challenges and opportunities. IEEE Acc. 2020, 8, 34564-34584. [CrossRef]

90. Chen, B.; Wan, J.; Shu, L.; Li, P.; Mukherjee, M.; Yin, B. Smart factory of industry 4.0: Key technologies, application case, and challenges. IEEE Acc. 2018, 6, 6505-6519. [CrossRef]

91. What is Industry 4.0 - the Industrial Internet of Things (IIoT)? Available online: https://www.epicor.com/en-in/resource-center/ articles/what-is-industry-4-0/ (accessed on 1 January 2021).

92. Andronie, M.; Lăzăroiu, G.; Iatagan, M.; Hurloiu, I.; Dijmărescu, I. Sustainable Cyber-Physical Production Systems in Big Data-Driven Smart Urban Economy: A Systematic Literature Review. Sustainability 2021, 13, 751. [CrossRef]

93. Marantos, C.; Siozios, K.; Soudris, D. A flexible decision-making mechanism targeting smart thermostats. IEEE Embed. Sys. Lett. 2017, 9, 105-108. [CrossRef]

94. Sivils, P.; Rieger, C.; Amarasinghe, K.; Manic, M. Integrated Cyber Physical Assessment and Response for Improved Resiliency. In The Internet of Things for Smart Urban Ecosystems; Springer: Cham, Switzerland, 2019; pp. 51-74. 
95. Marantos, C.; Siozios, K.; Soudris, D. Rapid Prototyping of Low-Complexity Orchestrator Targeting Cyber Physical Systems: The Smart-Thermostat Use case. IEEE Trans. Control Syst. Technol. 2020. [CrossRef]

96. Dos Reis, B.R.; Easton, Z.; White, R.R.; Fuka, D. A LoRa sensor network for monitoring pastured livestock location and activity. Trans. Anim. Sci. 2021, txab010. [CrossRef]

97. Morth, O.; Emmanouilidis, C.; Hafner, N.; Schadler, M. Cyber-physical systems for performance monitoring in production Intralogistics. Comput. Ind. Eng. 2020, 142, 106333. [CrossRef]

98. Cali, U.; Kuzlu, M.; Sharma, V.; Pipattanasomporn, M.; Catak, F.O. Internet of Predictable Things (IoPT) Framework to Increase Cyber-Physical System Resiliency. arXiv 2021, arXiv:2101.07816.

99. Sharma, R.; Parhi, S.; Shishodia, A. Industry 4.0 applications in agriculture: Cyber-physical agricultural systems (CPASs). In Advances in Mechanical Engineering; Springer: Singapore, 2021; pp. 807-813.

100. Verdouw, C.; Tekinerdogan, B.; Beulens, A.; Wolfert, S. Digital twins in smart farming. Agric. Syst. 2021, 189, 103046. [CrossRef]

101. Neethirajan, S. The role of sensors, big data and machine learning in modern animal farming. Sens. Bio-Sens. Res. 2020, 29, 100367-1-100367-8. [CrossRef]

102. Rugarolas, R.; Loftin, R.T.; Yang, P.; Roberts, D.L.; Sherman, B.; Bozkurt, A. Behavior recognition based on machine learning algorithms for a wireless canine machine interface. In Proceedings of the 2013 IEEE International Conference on Body Sensor Networks; IEEE: Cambridge, MA, USA, 2013; pp. 1-5.

103. Jukan, A.; Masip-Bruin, X.; Amla, N. Smart computing and sensing technologies for animal welfare: A systematic review. ACM Comput. Surv. 2017, 50, 10. [CrossRef]

104. Chung, Y.; Lee, J.; Oh, S.; Park, D.; Chang, H.H.; Kim, S. Automatic Detection of Cow's Oestrus in Audio Surveillance System. Asian-Australas. J. Anim. Sci. 2013, 26, 1030-1037. [CrossRef]

105. Alsouda, Y.; Pllana, S.; Kurti, A. IoT-based Urban Noise Identification Using Machine Learning: Performance of SVM, KNN, Bagging, and Random Forest. In Proceedings of the International Conference on Omni-Layer Intelligent Systems, Crete, Greece, 5-7 May 2019; ACM: New York, NY, USA, 2019; pp. 62-67.

106. Yang, K.; Liu, S.; Cai, L.; Yilmaz, Y.; Chen, P.Y.; Walid, A. Guest Editorial Special Issue on AI Enabled cognitive communication and networking for IoT. IEEE Internet Things J. 2019, 6, 1906-1910. [CrossRef]

107. Lin, Y.W.; Lin, Y.B.; Liu, C.Y. AItalk: A tutorial to implement AI as IoT devices. IET Netw. 2019, 8, 195-202. [CrossRef]

108. Ganz, F.; Puschmann, D.; Barnaghi, P.; Carrez, F. A practical evaluation of information processing and abstraction techniques for the IoT. IEEE Internet Things J. 2015, 2, 340-354. [CrossRef]

109. Mahdavinejad, M.S.; Rezvan, M.; Barekatain, M.; Adibi, P.; Barnaghi, P.; Sheth, A.P. Machine learning for IoT data analysis: A survey. Digit. Commun. Netw. 2018, 4, 161-175. [CrossRef]

110. Manogaran, G.; Shakeel, P.M.; Fouad, H.; Nam, Y.; Baskar, S.; Chilamkurti, N.; Sundarasekar, R. Wearable IoT Smart-Log Patch: An Edge Computing-Based Bay deep learning network system for multi access physical monitoring system. Sensors 2019, 19, 3030. [CrossRef]

111. Angin, P.; Anisi, M.H.; Goksel, F.; Gursoy, C.; Buyukgulcu, A. AgriLoRa: A Digital Twin Framework for Smart Agriculture. J. Wire. Mob. Netw. Ubiq. Comp. Dept. Appl. 2020, 11,77-96. [CrossRef]

112. Kumar, R. IoT and Deep Learning for Livestock Management. In Examining the Impact of Deep Learning and IoT on Multi-Industry Applications; IGI Global: Hershey, PA, USA, 2021; pp. 80-96.

113. Zgank, A. IoT-Based Bee Swarm Activity Acoustic Classification Using Deep Neural Networks. Sensors 2021, 21, 676. [CrossRef] [PubMed]

114. Ma, N.; Pan, L.; Chen, S.; Liu, B. NB-IoT Estrus Detection System of Dairy Cows Based on LSTM Networks. In Proceedings of the IEEE 31st Annual International Symposium on Personal, Indoor and Mobile Radio Communications, London, UK, 31 August 2020; IEEE: New York, NY, USA, 2020; pp. 1-5.

115. Larsen, M.L.; Wang, M.; Norton, T. Information Technologies for Welfare Monitoring in Pigs and Their Relation to Welfare Quality. Sustainability 2021, 13, 692. [CrossRef]

116. Silva, A.P.S.P.; de Freitas Costa, E.; de Silva, G.S.; Souza, C.K.; Schaefer, R.; da Silva Vaz, I., Jr.; Corbellini, L.G. Biosecurity practices associated with influenza: A virus seropre-valence in sows from southern Brazilian breeding herds. Prev. Vet. Med. 2019, 166, 1-7. [CrossRef]

117. Reusken, C.; van den Wijngaard, C.; van Beek, P.; Beer, M.; Bouwstra, R.; Godeke, G.J.; Isken, L.; Kerkhof, H.V.D.; Van Pelt, W.; Van Der Poel, W.; et al. Lack of evidence for zoonotic transmission of Schmallenberg virus. Emerg. Inf. Dis. 2012, 18, 1746. [CrossRef] [PubMed]

118. Veldhuis, A.; Mars, J.; Stegeman, A.; van Schaik, G. Changing surveillance objectives during the different phases of an emerging vector-borne disease outbreak: The Schmallenberg virus example. Prev. Vet. Med. 2019, 166, 21-27. [CrossRef]

119. Kwok, K.T.; Nieuwenhuijse, D.F.; Phan, M.V.; Koopmans, M.P. Virus metagenomics in farm animals: A systematic review. Viruses 2020, 12, 107. [CrossRef]

120. Mackenzie, J.S.; Smith, D.W. COVID-19: A novel zoonotic disease caused by a coronavirus from China: What we know and what we don't. Microbiol. Aust. 2020, 41, 45-50. [CrossRef] [PubMed]

121. Vayssade, J.A.; Arquet, R.; Bonneau, M. Automatic activity tracking of goats using drone camera. Comput. Electron. Agric. 2019, 162, 767-772. [CrossRef] 
122. Caldara, F.R.; Dos Santos, L.S.; Machado, S.T.; Moi, M.; Nääs, I.D.A.; Foppa, L.; Garcia, R.G.; Santos, R.D.K.S.D. Piglets' Surface Temperature Change at Different Weights at Birth. Asian-Australas. J. Anim. Sci. 2014, 27, 431. [CrossRef] [PubMed]

123. Mellor, D.J. Updating animal welfare thinking: Moving beyond the "Five Freedoms" towards "a Life Worth Living". Animals 2016, 6, 21. [CrossRef] [PubMed]

124. Blokhuis, H.; Veissier, I.; Miele, M.; Jones, B. Safeguarding farm animal welfare. Sustain. Certif. Schemes Agric. Nat. Resour. Sect. 2019, 137. [CrossRef]

125. Bowling, M.B.; Pendell, D.L.; Morris, D.L.; Yoon, Y.; Katoh, K.; Belk, K.E.; Smith, G.C. Identification and traceability of cattle in selected countries outside of N. America. Prof. Anim. Sci. 2008, 24, 287-294. [CrossRef]

126. Zhao, J.; Xu, Z.; You, X.; Zhao, Y.; He, W.; Zhao, L.; Chen, A.; Yang, S. Genetic traceability practices in a large-size beef company in China. Food Chem. 2019, 277, 222-228. [CrossRef] [PubMed]

127. Review of Livestock Identification and Traceability in the UK. Available online: http:/ / randd.defra.gov.uk/Document.aspx? Document=11417_IdentificationandTraceabilityFinal.pdf (accessed on 2 August 2019).

128. Kumar, S.; Singh, S.K. Cattle Recognition: A New Frontier in Visual Animal Biometrics Research. Proc. Natl. Acad. Sci. India Sect. A Phys. Sci. 2020, 90, 689-708. [CrossRef]

129. Fernandez-Carames, T.; Fraga-Lamas, P.; Suarez-Albela, M.; Castedo, L. Reverse engineering and security evaluation of commercial tags for RFID-based IoT applic. Sensors 2017, 17, 28. [CrossRef] [PubMed]

130. Fenil, E.; Manogaran, G.; Vivekananda, G.N.; Thanjaivadivel, T.; Jeeva, S.; Ahilan, A. Real time violence detection framework for football stadium comprising of big data analysis and deep learning through bidirectional LSTM. Comput. Netw. 2019, 151, 191-200.

131. Kumar, S.; Pandey, A.; Satwik, K.S.R.; Kumar, S.; Singh, S.K.; Singh, A.K.; Mohan, A. Deep learning framework for recognition of cattle using muzzle point image pattern. Measurement 2018, 116, 1-17. [CrossRef]

132. Kumar, S.; Singh, S.K. Monitoring of pet animal in smart cities using animal biometrics. Future Gener. Comput. Syst. 2018, 83, 553-563. [CrossRef]

133. Tang, S.; Shelden, D.R.; Eastman, C.M.; Pishdad-Bozorgi, P.; Gao, X. A review of building inf. modeling and IoT devices integration: Present status and future trends. Autom. Constr. 2019, 101, 127-139. [CrossRef]

134. Mutlag, A.A.; Ghani, M.K.A.; Arunkumar, N.A.; Mohamed, M.A.; Mohd, O. Enabling technologies for fog computing in healthcare IoT sys. Future Gener. Comput. Syst. 2019, 90, 62-78. [CrossRef]

135. Cui, M.; Fei, Y.; Liu, Y. A Survey on Secure Deployment of Mobile Services in Edge Computing. Secur. Commun. Netw. 2021, 1-8. [CrossRef]

136. Danneels, E. Disruptive technology reconsidered: A critique and research agenda. J. Prod. Innov. Mgt. 2004, 21, 246-258. [CrossRef]

137. Coccia, M. General sources of general purpose tech. in complex societies: Theory of global leadership-driven innovation, warfare and human dev. Technol. Soc. 2015, 42, 199-226. [CrossRef]

138. Teece, D.J. Profiting from innovation in the digital economy: Enabling technologies, standards, and licensing models in the wireless world. Res. Pol. 2018, 47, 1367-1387. [CrossRef]

139. Atzori, L.; Iera, A.; Morabito, G. The IoT: A survey. Comput. Netw. 2010, 54, 2787-2805. [CrossRef]

140. GulfBusiness: The Role of Big Data in Unlocking the True Value of IoT. Available online: https://gulfbusiness.com/the-role-ofbig-data-in-unlocking-the-true-value-of-iot/ (accessed on 13 December 2020).

141. Natsiavas, P.; Rasmussen, J.; Voss-Knude, M.; Votis, K.; Coppolino, L.; Campegiani, P.; Cano, I.; Marí, D.; Faiella, G.; Clemente, F.; et al. Comprehensive user requirements engineering methodology for secure and interoperable health data exchange. BMC Med Inform. Decis. Mak. 2018, 18, 85. [CrossRef] [PubMed]

142. Alonso, R.S.; Sittón-Candanedo, I.; García, O.; Prieto, J.; Rodríguez-González, S. An intelligent Edge-IoT platform for monitoring livestock and crops in a dairy farming scenario. Ad Hoc Netw. 2020, 98, 102047. [CrossRef]

143. Pillai, R.; Sivathanu, B. Adoption of IoT in the agriculture industry deploying the BRT framework. Benchmarking Int. J. 2020, 27, 1341-1368. [CrossRef]

144. Farooq, M.S.; Riaz, S.; Abid, A.; Abid, K.; Naeem, M.A. A Survey on the Role of IoT in Agriculture for the Implementation of Smart Farming. IEEE Access 2019, 7, 156237-156271. [CrossRef]

145. Farooq, M.S.; Riaz, S.; Abid, A.; Umer, T.; Zikria, Y.B. Role of IoT Technology in Agriculture: A Systematic Literature Review. Electronics 2020, 9, 319. [CrossRef]

146. Boursianis, A.D.; Papadopoulou, M.S.; Diamantoulakis, P.; Liopa-Tsakalidi, A.; Barouchas, P.; Salahas, G.; Karagiannidis, G.; Wan, S.; Goudos, S.K. IoT and Agricultural Unmanned Aerial Vehicles (UAVs) in Smart Farming: A Comprehensive Review. Internet Things 2020, 100187. [CrossRef]

147. Dupont, C.; Vecchio, M.; Pham, C.; Diop, B.; Dupont, C.; Koffi, S. An Open IoT Platform to Promote Eco-Sustainable Innovation in Western Africa: Real Urban and Rural Testbeds. Wirel. Commun. Mob. Comput. 2018, 2018, 1-17. [CrossRef]

148. Verdouw, C.; Sundmaeker, H.; Tekinerdogan, B.; Conzon, D.; Montanaro, T. Architecture framework of IoT-based food and farm systems: A multiple case study. Comput. Electron. Agric. 2019, 165, 104939. [CrossRef]

149. Adi, E.; Anwar, A.; Baig, Z.; Zeadally, S. Machine learning and data analytics for the IoT. Neural Comput. Appl. 2020, 32, 16205-16233. [CrossRef] 
150. Bilal, M.; Oyedele, L.O.; Qadir, J.; Munir, K.; Ajayi, S.O.; Akinade, O.O.; Owolabi, H.A.; Alaka, H.A.; Pasha, M. Big Data in the construction industry: A review of present status, opportunities, and future trends. Adv. Eng. Inform. 2016, 30, 500-521. [CrossRef]

151. Otles, S.; Sakalli, A. Industry 4.0: The Smart Factory of the Future in Beverage Industry. In Production and Management of Beverages; Woodhead Publishing: Sawston, UK, 2019; pp. 439-469.

152. Bayne, K.; Turner, P.V. Animal Welfare Standards and International Collaborations. ILAR J. 2019, 1-9. [CrossRef]

153. Ayodele, T.O.; Oladokun, T.T.; Kajimo-Shakantu, K. Employability skills of real estate graduates in Nigeria: A skill gap analysis. J. Facil. Manag. 2020, 18, 297-323. [CrossRef] 